\title{
Origin of apparent period variations in eclipsing post-common-envelope binaries ${ }^{\star}$
}

\author{
M. Zorotovic ${ }^{1}$ and M. R. Schreiber ${ }^{1,2}$ \\ 1 Departamento de Física y Astronomía, Facultad de Ciencias, Universidad de Valparaíso, Valparaíso, Chile \\ e-mail: mzorotovic@dfa.uv.cl \\ 2 Millennium Nucleus "Protoplanetary Disks in ALMA Early Science”, Universidad de Valparaíso, Valparaíso, Chile
}

Received 31 August 2012 / Accepted 6 November 2012

\section{ABSTRACT}

\begin{abstract}
Context. Apparent period variations detected in several eclipsing, close-compact binaries are frequently interpreted as being caused by circumbinary giant planets. This interpretation raises the question of the origin of the potential planets that must have either formed in the primordial circumbinary disk, together with the host binary star, and survived its evolution into a close-compact binary or formed in a post-common-envelope circumbinary disk that remained bound to the post-common-envelope binary (PCEB).

Aims. Here we combine current knowledge of planet formation and the statistics of giant planets around primordial and evolved binary stars with the theory of close-compact binary star evolution aiming to derive new constraints on possible formation scenarios. Methods. We compiled a comprehensive list of observed eclipsing PCEBs, estimated the fraction of systems showing apparent period variations, reconstructed the evolutionary history of the PCEBs, and performed binary population models of PCEBs to characterize their main sequence binary progenitors. We reviewed the currently available constraints on the fraction of PCEB progenitors that host circumbinary giant planets.

Results. We find that the progenitors of PCEBs are very unlikely to be frequent hosts of giant planets $(\$ 10$ per cent), while the frequency of PCEBs with observed apparent period variations is very high ( $\sim 90$ per cent).

Conclusions. The variations in eclipse timings measured in eclipsing PCEBs are probably not caused by first-generation planets that survived common-envelope evolution. The remaining options for explaining the observed period variations are second-generation planet formation or perhaps variations in the shape of a magnetically active secondary star. We suggest observational tests for both options.
\end{abstract}

Key words. binaries: close - binaries: eclipsing - planetary systems - planets and satellites: formation

\section{Introduction}

The discovery of extrasolar planets (exoplanets) in the nineties and the subsequent detection of more than 700 exoplanets until now (Schneider et al. 2011) has taken us much closer toward answering fundamental questions, such as how planets form from circumstellar material and what the fate of planetary systems is when their host stars evolve into giant stars and compact objects. Especially that exoplanets have been detected in rather unexpected exotic environments might go further pave our way towards solving these important questions.

Pulsar timings have led to detecting the first confirmed exoplanet orbiting the pulsar PSR B1257+12 (Wolszczan \& Frail 1992; Wolszczan 1994), and continued monitoring of the system has revealed the existence of a second and a third planet (Wolszczan 1994), even the possibility of a fourth one (Wolszczan 1997). The first exoplanet around a solar-type star, a Jupiter-mass companion to the star 51 Pegasi, was identified and confirmed thanks to radial velocity variations (Mayor \& Queloz 1995). This finding generated large radial velocity surveys for exoplanets, and meanwhile more than 600 planets around main sequence (MS) stars have been identified using this method. Circumbinary exoplanets around binary stars composed of two MS stars (MS+MS binaries) were predicted long ago, but only

\footnotetext{
* Appendix A is available in electronic form at http://www. aanda.org
}

recently have the first six detections been possible (Doyle et al. 2011; Welsh et al. 2012; Orosz et al. 2012a,b).

Among the perhaps most unexpected potential planet detections are those derived from apparent period variations (or eclipse time variations), frequently measured in eclipsing, closecompact binary stars containing a white dwarf (WD) or hot subdwarf B (sdB) primary star and a low-mass star companion. The first of these substellar third bodies, a brown dwarf (BD), was announced roughly a decade ago around the post-commonenvelope binary (PCEB) V471 Tau (Guinan \& Ribas 2001). At present, detection of substellar and mostly planetary circumbinary companions has been claimed for a dozen eclipsing, closecompact binaries containing WD or hot sdB primary stars (see Beuermann et al. 2010, for the most convincing example). While the parameters derived for these potential circumbinary giant planets have to be considered with extreme caution, it is true that a large number of PCEBs show apparent period variations that might be explained by changes in the light travel time caused by a circumbinary giant planet. We present an observational census of eclipsing, close-compact binaries and eclipse timing measurements in the appendix.

If confirmed, PCEBs as hosts of giant planets are particularly interesting because the binary host star passed through a special evolutionary phase: common-envelope (CE) evolution. As outlined by Paczyński (1976) and described in much more detail by Webbink (1984) CE evolution occurs once the initially 
more massive star (the primary) evolves. If it fills its Roche lobe during one of the giant phases, when it has a deep convective envelope, dynamically unstable mass transfer to the less massive component (the secondary) begins. The secondary is not able to adjust its structure on the mass-transfer time scale. The material lost by the primary therefore initially fills the Roche lobe of the secondary and then grows to form a noncorotating CE that surrounds the core of the giant and the secondary star. Owing to drag forces within the envelope, orbital energy is extracted from the binary and transferred to the envelope, which dramatically reduces the separation between the core of the primary and the secondary star in a spiraling-in process, until the envelope becomes unbound and is ejected from the binary. The remaining system is a PCEB consisting of the core of the primary (a compact object) and an MS companion, in a close but detached orbit. The short duration of the CE phase $\left(\$ 10^{3} \mathrm{yr}\right)$ means that the mass of the secondary star is assumed to remain constant (Hjellming \& Taam 1991), and the mass of the compact object should be equal to the mass of the core of the giant at the onset of mass transfer (for more details of CE evolution see, e.g., Iben \& Livio 1993; Webbink 2008; Zorotovic et al. 2010).

The special evolution of the host binary stars implies that the claimed circumbinary planets must have either survived the dramatic evolution of the host binary star or must have formed as a consequence of this evolution. Which of these two spectacular scenarios might have taken place in PCEBs with candidate circumbinary planets is currently a completely open question (see, e.g., the discussion in Beuermann et al. 2010). Answering it based on hydrodynamic simulations of CE evolution, such as those presented by Ricker \& Taam (2012), is currently impossible, because CE evolution is still relatively uncertain even without an embedded planet.

In this paper, we present the results of binary population synthesis simulations and reconstruct the evolutionary history of observed eclipsing PCEBs to characterize their MS+MS binary progenitors. Reviewing what is known about circumbinary planets and circumbinary planet formation around such MS+MS binaries, we conclude that the apparent period variations observed in PCEBs are very unlikely to be caused by giant planets that survived $\mathrm{CE}$ evolution, and we discuss alternative explanations. We start with a review of the observed sample of eclipsing PCEBs and the detected apparent period variations.

\section{Giant planets around compact binaries?}

In the past few years, it has been suggested that there are giant planets around several eclipsing, close-compact binaries to explain observed variations of eclipse timings. These discoveries have become possible thanks to both the increase in the number of known eclipsing systems in recent years and the improvement in the accuracy of the observed light curves and derived eclipse timings.

Almost a decade ago, Schreiber \& Gänsicke (2003) analyzed 30 detached PCEBs when only 11 eclipsing systems, including the prototype V471 Tau, were known. The SDSS and intensive follow-up observations of WD+MS binaries, as well as the Catalina Realtime Transient Survey, significantly increased the number of known eclipsing, detached PCEBs. Table 1 lists the orbital parameters of 56 known eclipsing, detached PCEBs and separates systems with WD and sdB primary stars. We excluded eclipsing PCEBs that are in the center of a planetary nebula, because no accurate eclipse timings are available for those systems. A brief review of the observational history of the 56 eclipsing PCEBs can be found in the appendix.
Currently, 13 eclipsing, detached PCEBs consisting of an $\mathrm{sdB}$ (or sdOB) primary and a MS or BD companion are known. For an amazing fraction of $\sim 38$ per cent, apparent period changes that might be caused by a third substellar body have been measured (systems in bold in Table 1). Even more dramatic, as shown in the appendix, almost all systems that have been intensively followed up for more than $\sim 5$ years show period variations that might indicate the presence of a third body, with AA Dor the only exception (Kilkenny 2011).

The sample of known eclipsing, detached PCEBs with a WD primary contains 43 systems. For four of them, thirdbody detections using eclipse timing measurements have been claimed. Very few eclipse timings have been published for almost all other systems, mostly because the systems have been discovered fairly recently. In addition, several early eclipse timings are not reliable. This means that, as in the case of $\mathrm{sdB}+\mathrm{MS}$ eclipsing binaries, all systems with accurate eclipse timings (error $\lesssim 10 \mathrm{~s}$ ) covering $\gtrsim 5$ years show apparent period changes that may indicate there is a third circumbinary object.

In the past few years, apparent period variations associated to the presence of a third body have also been observed for three cataclysmic variables (CVs): UZFor, HU Aqr, and DPLeo. $\mathrm{CVs}$ are PCEBs that evolved into a semi-detached configuration where a Roche lobe-filling MS star (or a BD) transfers mass on the WD. Eclipsing CVs were discovered more than a century ago (see, e.g., Pogson 1857), and up to now there are almost 200 eclipsing systems among the $\sim 1000 \mathrm{CVs}$ listed in the catalog of Ritter \& Kolb (2003, V7.16). A complete discussion of all these systems is beyond the scope of this paper, but for completeness, we list the parameters of the three CVs with claimed planet detections in Table 1 and briefly review the observational history of these three systems in the appendix.

The orbital parameters obtained with the best fit available for the claimed planets are listed in Table 2. It should be noticed that, at present, not a single set of orbital parameters has been confirmed by new measurements. In addition, some of the claimed planetary systems seem to be unstable (see, e.g., Horner et al. 2011; Hinse et al. 2012).

Still, the large fraction of PCEBs that show apparent period variations is intriguing. Nine out of ten detached PCEBs, which corresponds to a fraction of $90 \pm 9$ per cent, with accurate eclipse timing measurements covering $\sim 5$ years show clear variations that might indicate the presence of a third body. If these potential planets have formed prior to $\mathrm{CE}$ evolution, a similarly large fraction of the MS+MS progenitor binaries of PCEBs must also host giant planets. To evaluate whether this might indeed be the case, we need to derive the orbital and stellar parameters of the PCEB progenitors.

\section{Binary population simulation}

To characterize the progenitors of PCEBs we performed binary population studies of PCEBs containing a WD or an sdB primary. We complement our simulations by reconstructing the evolutionary history of the observed sample using the code described in Zorotovic et al. (2011a).

Binary population studies of WD+MS PCEBs have been previously performed by several authors (see, e.g., de Kool \& Ritter 1993; Willems \& Kolb 2004; Politano \& Weiler 2006, 2007; Davis et al. 2010), and recently, Clausen et al. (2012) have simulated the population of $\mathrm{sdB}+\mathrm{MS}$ binaries. We here present the first population simulation of PCEBs including both systems with WD and sdB primary stars. In addition, it is the first time 
M. Zorotovic and M. R. Schreiber: Origin of apparent period variations in PCEBs

Table 1. Orbital parameters for the currently known eclipsing PCEBs.

\begin{tabular}{|c|c|c|c|c|c|}
\hline System & Alt. Name & $\begin{array}{c}P_{\text {orb }} \\
{[\mathrm{d}]}\end{array}$ & $\begin{array}{c}M_{1} \\
{\left[M_{\odot}\right]}\end{array}$ & $\begin{array}{c}M_{2} \\
{\left[M_{\odot}\right]}\end{array}$ & References \\
\hline \multicolumn{6}{|c|}{ Detached sdB+MS/BD PCEBs } \\
\hline HW Vir & 2MJ1244-0840 & 0.11671955 & $0.485 \pm 0.013$ & $0.142 \pm 0.004$ & 1,2 \\
\hline HS 0705+6700 & $2 M J 0710+6655$ & 0.095646625 & 0.483 & 0.134 & 3,4 \\
\hline HS 2231+2441 & $2 M J 2234+2456$ & 0.110588 & $0.47:$ & 0.075: & 5 \\
\hline NSVS 14256825 & 2M J2020+0437 & 0.11037423 & $0.419 \pm 0.070$ & $0.109 \pm 0.023$ & 6 \\
\hline NY Vir & PG 1336-018 & 0.101015967 & $0.459 \pm 0.005$ & $0.122 \pm 0.001$ & $7,8,9$ \\
\hline $2 \mathrm{M} 1938+4603$ & NSVS 05629361 & 0.1257653 & $0.48 \pm 0.03$ & $0.12 \pm 0.01$ & 10 \\
\hline NSVS 07826247 & CSS06833 & 0.16177042 & $0.376 \pm 0.055$ & $0.113 \pm 0.017$ & 11 \\
\hline BUL-SC16 335 & 2M J1809-2641 & 0.12505028 & $0.5:$ & $0.16:$ & 12 \\
\hline PG $1621+4737$ & $2 \mathrm{MJ} 1622+4730$ & 0.075 & - & - & 13 \\
\hline \multirow{2}{*}{ SDSS J0820+0008 } & GSC 0196.0617 & 0.097 & $\sim 0.25$ & $0.045 \pm 0.03$ & 14,15 \\
\hline & & & $\sim 0.47$ & $0.068 \pm 0.03$ & 15 \\
\hline ASAS 10232 & $2 \mathrm{M} \mathrm{J} 1023-3736$ & 0.13927 & $0.461 \pm 0.051$ & $0.157 \pm 0.017$ & 16 \\
\hline AA Dor & LB 3459 & 0.261539736 & $0.471 \pm 0.005$ & $0.0788_{-0.0063}^{+0.0075}$ & 17,18 \\
\hline EC 10246-2707 & & 0.118507993 & $0.45 \pm 0.17$ & $0.12 \pm 0.05$ & 19 \\
\hline \multicolumn{6}{|c|}{ Detached WD+MS PCEBs } \\
\hline NNSer & 2MJ1552+1254 & 0.13008014 & $0.535 \pm 0.012$ & $0.111 \pm 0.004$ & 20,21 \\
\hline V471 Tau & 2M J0350+1714 & 0.52118343 & $0.84 \pm 0.05$ & $0.93 \pm 0.07$ & 22,23 \\
\hline QS Vir & EC 13471-1258 & 0.1507575 & $0.78 \pm 0.040$ & $0.430 \pm 0.040$ & 24 \\
\hline RR Cae & 2M J0421-4839 & 0.30370363 & $0.440 \pm 0.022$ & $0.183 \pm 0.013$ & 25 \\
\hline DE Cvn & RXJ1326.9+4532 & 0.364139315 & $0.51_{-0.02}^{+0.06}$ & $0.41 \pm 0.06$ & 26,27 \\
\hline GK Vir & SDSS J1415+0117 & 0.344330833 & $0.564 \pm 0.014$ & $0.116 \pm 0.003$ & 28 \\
\hline RX J2130.6+4710 & $2 \mathrm{M} \mathrm{J} 2130+4710$ & 0.52103562 & $0.554 \pm 0.017$ & $0.555 \pm 0.023$ & 29 \\
\hline SDSS J0110+1326 & WD $0107+131$ & 0.332687 & $0.47 \pm 0.02$ & $0.255-0.380$ & 30 \\
\hline SDSS J0303+0054 & & 0.1344377 & $0.878-0.946$ & $0.224-0.282$ & 30 \\
\hline SDSS J0857+0342 & CSS03170 & 0.06509654 & $0.51 \pm 0.05$ & $0.09 \pm 0.01$ & 31 \\
\hline SDSS J1210+3347 & & 0.12448976 & $0.415 \pm 0.010$ & $0.158 \pm 0.006$ & 32 \\
\hline SDSS J1212-0123 & & 0.3358711 & $0.439 \pm 0.002$ & $0.273 \pm 0.002$ & 27 \\
\hline SDSS J1435+3733 & & 0.125631 & $0.48-0.53$ & $0.190-0.246$ & 30 \\
\hline SDSS J1548+4057 & & 0.1855177 & $0.614-0.678$ & $0.146-0.201$ & 30 \\
\hline CSS06653 & SDSS J1329+1230 & 0.08096625 & $0.350 \pm 0.081$ & - & 33,34 \\
\hline CSS07125 & SDSS J1410-0202 & 0.363497 & $0.470 \pm 0.055$ & $0.380 \pm 0.012$ & 34,35 \\
\hline CSS080408 & SDSS J1423+2409 & 0.3820040 & $0.410 \pm 0.024$ & $0.255 \pm 0.040$ & 34,35 \\
\hline CSS080502 & SDSS J0908+0604 & 0.14943807 & $0.370 \pm 0.018$ & $0.319 \pm 0.061$ & 33,34 \\
\hline CSS09704 & SDSS J2208-0115 & 0.1565057 & 0.37 & - & 35 \\
\hline CSS09797 & SDSS J1456+1611 & 0.229120 & $0.370 \pm 0.016$ & $0.196 \pm 0.043$ & 34,35 \\
\hline CSS21357 & SDSS J1348+1834 & 0.2484 & $0.590 \pm 0.017$ & $0.319 \pm 0.061$ & 34,35 \\
\hline CSS21616 & SDSS J1325+2338 & 0.1949589 & - & - & 33 \\
\hline CSS25601 & SDSS J1244+1017 & 0.227856 & $0.400 \pm 0.026$ & $0.319 \pm 0.061$ & 34,35 \\
\hline CSS38094 & SDSS J0939+3258 & 0.3309896 & $0.520 \pm 0.026$ & $0.319 \pm 0.061$ & 33,34 \\
\hline CSS40190 & SDSS J0838+1914 & 0.13011232 & $0.390 \pm 0.035$ & $0.255 \pm 0.040$ & 33,34 \\
\hline CSS41631 & SDSS J0957+2342 & 0.15087074 & $0.430 \pm 0.025$ & $0.431 \pm 0.108$ & 33,34 \\
\hline WD $1333+005$ & SDSS J1336+0017 & 0.1219587 & - & - & 33 \\
\hline PTFEB 11.441 & PTF1 J004546.0+415030.0 & 0.3587 & $0.51 \pm 0.09$ & $0.35 \pm 0.05$ & 36 \\
\hline PTFEB28.235 & PTF1 J015256.6+384413.4 & 0.3861 & $0.65 \pm 0.11$ & $0.35 \pm 0.05$ & 36 \\
\hline PTFEB28.852 & PTF1 J015524.7+373153.8 & 0.4615 & $0.52 \pm 0.05$ & $0.35 \pm 0.05$ & 36 \\
\hline KIC-10544976 & USNO-B1.0 1377-0415424 & 0.35046872 & $0.61 \pm 0.04$ & $0.39 \pm 0.03$ & 37 \\
\hline SDSS J0821+4559 & & 0.50909 & $0.66 \pm 0.05$ & $0.431 \pm 0.108$ & 34,38 \\
\hline SDSS J0927+3329 & & 2.30822 & $0.59 \pm 0.05$ & $0.380 \pm 0.012$ & 34,38 \\
\hline SDSS J0946+2030 & & 0.252861219 & $0.62 \pm 0.10$ & $0.255 \pm 0.040$ & 34,38 \\
\hline SDSS J0957+3001 & & 1.92612 & $0.42 \pm 0.05$ & $0.380 \pm 0.012$ & 34,38 \\
\hline SDSS J1021+1744 & & 0.14035907 & $0.50 \pm 0.05$ & $0.319 \pm 0.061$ & 34,38 \\
\hline SDSS J1028+0931 & & 0.23502576 & $0.42 \pm 0.04$ & $0.380 \pm 0.012$ & 34,38 \\
\hline
\end{tabular}


Table 1. continued.

\begin{tabular}{llcccl}
\hline \hline System & Alt. Name & $\begin{array}{c}P_{\text {orb }} \\
{[\mathrm{d}]}\end{array}$ & $\begin{array}{c}M_{1} \\
{\left[M_{\odot}\right]}\end{array}$ & $\begin{array}{c}M_{2} \\
{\left[M_{\odot}\right]}\end{array}$ & References \\
& & 0.1251621 & $0.34 \pm 0.07$ & $0.255 \pm 0.040$ & 34,38 \\
\hline SDSS J1057+1307 & & 0.09007 & $0.45 \pm 0.06$ & $0.196 \pm 0.043$ & 34,38 \\
SDSS J1223-0056 & & 0.216322132 & - & $0.319 \pm 0.061$ & 34,38 \\
SDSS J1307+2156 & & 0.1917902 & $0.49 \pm 0.04$ & $0.255 \pm 0.040$ & 34,38 \\
SDSS J1408+2950 & & 0.167509 & $0.36 \pm 0.04$ & $0.380 \pm 0.012$ & 34,38 \\
SDSS J1411+1028 & & 0.14445648 & $0.45 \pm 0.06$ & $0.319 \pm 0.061$ & 34,38 \\
SDSS J2235+1428 & & & & & \\
\hline CVs & & $\mathbf{0 . 0 8 7 8 6 5 4 2}$ & $\sim \mathbf{0 . 7 1}$ & $\sim \mathbf{0 . 1 4}$ & 39,40 \\
UZ For & 2M J0335-2544 & $\mathbf{0 . 0 8 6 8 2 0 4 1}$ & $\mathbf{0 . 8 0} \pm \mathbf{0 . 0 4}$ & $\mathbf{0 . 1 8} \pm \mathbf{0 . 0 6}$ & 41,42 \\
HU Aqr & 2M J2107-0517 & $\mathbf{0 . 1 4 :}$ & 43,44 \\
DP Leo & RX J2107.9-0518 & $\mathbf{0 . 0 6 2 3 6 2 8 6}$ & $\mathbf{1 . 2 :}$ & $\mathbf{0 . 1 4}$ & \\
& & & $\mathbf{0 . 6 :}$ & $\mathbf{0 . 0 9 :}$ & 45 \\
\hline
\end{tabular}

Notes. Systems with suspected planets are in bold. We distinguish between detached systems containing sdB primaries, detached systems with WD primaries, and CVs. For the case of CVs we only list those with suspected planets (see Sect. 2 for details). Very uncertain values are followed by “.”. It generally means that the mass was assumed and not derived. Systems with suspected planets are in bold. We distinguish between detached systems containing sdB primaries, detached systems with WD primaries, and CVs. For the case of CVs we only list those with suspected planets (see Sect. 2 for details).

References. (1) Lee et al. (2009); (2) Beuermann et al. (2012b); (3) Drechsel et al. (2001); (4) Beuermann et al. (2012a); (5) Østensen et al. (2007); (6) Almeida et al. (2012b); (7) Vučković et al. (2007); (8) Charpinet et al. (2008); (9) Qian et al. (2012b); (10) Østensen et al. (2010); (11) For et al. (2010); (12) Polubek et al. (2007); (13) Geier et al. (2010); (14) Geier et al. (2012); (15) Geier et al. (2011b); (16) Schaffenroth et al. (2011); (17) Kilkenny (2011); (18) Klepp \& Rauch (2011); (19) Barlow et al. (2012); (20) Parsons et al. (2010a); (21) Beuermann et al. (2010); (22) O'Brien et al. (2001); (23) Kundra \& Hric (2011); (24) O'Donoghue et al. (2003); (25) Maxted et al. (2007); (26) van den Besselaar et al. (2007); (27) Parsons et al. (2010b); (28) Parsons et al. (2012b); (29) Maxted et al. (2004); (30) Pyrzas et al. (2009); (31) Parsons et al. (2011a); (32) Pyrzas et al. (2012); (33) Backhaus et al. (2012); (34) Rebassa-Mansergas et al. (2012); (35) Drake et al. (2010); (36) Law et al. (2012); (37) Almenara et al. (2012); (38) Parsons et al. (2012a); (39) Bailey \& Cropper (1991); (40) Potter et al. (2011); (41) Schwarz et al. (2009); (42) Schwope et al. (2011); (43) Pandel et al. (2002); (44) Beuermann et al. (2011); (45) Schwope et al. (2002).

Table 2. Best fits of the orbital parameters for the currently claimed planets around eclipsing PCEBs.

\begin{tabular}{|c|c|c|c|c|c|c|}
\hline Name & $\begin{array}{c}M \sin (i) \\
{[\mathrm{Mj}]}\end{array}$ & $\begin{array}{c}P \\
{[\mathrm{yr}]}\end{array}$ & $\begin{array}{c}a \sin (i) \\
{[\mathrm{AU}]}\end{array}$ & $e$ & Ref. & Notes \\
\hline HW Vir c & $14.3 \pm 1.0$ & $12.7 \pm 0.2$ & $4.69 \pm 0.06$ & $0.40 \pm 0.10$ & 1 & \\
\hline HW Vir d & $30-120$ & $55 \pm 15$ & $12.8 \pm 0.2$ & $0.05:$ & 1 & $*$ \\
\hline HS0705+6700 c & $31.5 \pm 1.0$ & $8.41 \pm 0.05$ & 3.52 & $0.38 \pm 0.05$ & 2 & $*$ \\
\hline HS2231+2441 c & $13.94 \pm 2.20$ & 15.7 & $\sim 5.16$ & - & 3 & \\
\hline NSVS14256825 c & $2.8 \pm 0.3$ & $3.49 \pm 0.21$ & $1.9 \pm 0.3$ & $0.00 \pm 0.08$ & 4 & \\
\hline NSVS14256825 d & $8.0 \pm 0.8$ & $6.86 \pm 0.25$ & $2.9 \pm 0.6$ & $0.52 \pm 0.06$ & 4 & \\
\hline NY Vir c & $2.3 \pm 0.3$ & 7.9 & $3.3 \pm 0.8$ & - & 5 & \\
\hline NY Vir d & 2.5: & $>15$ & $\gtrsim 5.08$ & - & 5 & \\
\hline NN Ser c & $6.91 \pm 0.54$ & $15.50 \pm 0.45$ & $5.38 \pm 0.20$ & 0.0 & 6 & \\
\hline NN Ser d & $2.28 \pm 0.38$ & $7.75 \pm 0.35$ & $3.39 \pm 0.10$ & $0.20 \pm 0.02$ & 6 & \\
\hline V471 Tau c & $46-111$ & $33.2 \pm 0.2$ & $\sim 12.6-12.8$ & $0.26 \pm 0.02$ & 7 & $*$ \\
\hline QS Vir c & 9.01 & 14.4 & $\sim 6.32$ & 0.62 & 8 & \\
\hline QS Vir d & 56.59 & 16.99 & $\sim 7.15$ & 0.92 & 8 & * \\
\hline RR Cae c & $4.2 \pm 0.4$ & $11.9 \pm 0.1$ & $5.3 \pm 0.6$ & 0 & 9 & \\
\hline UZ For c & $6.3 \pm 1.5$ & $16+3$ & $5.9 \pm 1.4$ & $0.04 \pm 0.05$ & 10 & \\
\hline UZ For d & $7.7 \pm 1.2$ & $5.25 \pm 0.25$ & $2.8 \pm 0.5$ & $0.05 \pm 0.05$ & 10 & \\
\hline HU Aqr c & 7.1 & $9.00 \pm 0.05$ & 4.30 & $0.13 \pm 0.04$ & 11 & \\
\hline DP Leo c & $6.05 \pm 0.47$ & $28.01 \pm 2.00$ & $8.19 \pm 0.39$ & $0.39 \pm 0.13$ & 12 & \\
\hline
\end{tabular}

Notes. ${ }^{(*)}$ The claimed third body is more consistent with a BD than with a planet. Very uncertain values are followed by ":".

References. (1) Beuermann et al. (2012b); (2) Beuermann et al. (2012a); (3) Qian et al. (2010c); (4) Almeida et al. (2012a); (5) Qian et al. (2012b); (6) Beuermann et al. (2010); (7) Kundra \& Hric (2011); (8) Almeida \& Jablonski (2011); (9) Qian et al. (2012a); (10) Potter et al. (2011); (11) Goździewski et al. (2012); (12) Beuermann et al. (2011).

that the present-day population of PCEBs is simulated with a low value of the $\mathrm{CE}$ efficiency, including a fraction of the recombination energy, based on the results of Zorotovic et al. (2010). A full parameter study will be presented elsewhere.

\subsection{Initial conditions and assumptions}

We use a Monte Carlo code to generate an initial population of $10^{8} \mathrm{MS}+\mathrm{MS}$ binaries. The mass of the primary star 
is distributed according to the initial mass function (IMF) of Kroupa et al. (1993); i.e., the number of primaries with masses in the range $\mathrm{d} M_{1}$ is given by $\mathrm{d} N \propto f\left(M_{1}\right) \mathrm{d} M_{1}$ where $f\left(M_{1}\right)$ is given by

$$
f\left(M_{1}\right)= \begin{cases}0 & M_{1} / M_{\odot}<0.1 \\ 0.29056 M_{1}^{-1.3} & 0.1 \leq M_{1} / M_{\odot}<0.5 \\ 0.15571 M_{1}^{-2.2} & 0.5 \leq M_{1} / M_{\odot}<1.0 \\ 0.15571 M_{1}^{-2.7} & 1.0 \leq M_{1} / M_{\odot} .\end{cases}
$$

The mass of the secondary is assumed to be distributed according to a flat initial-mass-ratio distribution, i.e. $n(q)=$ constant, where $q=M_{2} / M_{1}$. The initial orbital separation $a_{\text {i follows the }}$ distribution

$$
h\left(a_{\mathrm{i}}\right)= \begin{cases}0 & a_{\mathrm{i}} / R_{\odot}<3 \text { or } a_{\mathrm{i}} / R_{\odot}>10^{6}, \\ 0.078636 a_{\mathrm{i}}^{-1} & 3 \leq a_{\mathrm{i}} / R_{\odot} \leq 10^{6}\end{cases}
$$

(Davis et al. 2008). We also assign a "born time" ( $\left.t_{\text {born }}\right)$ to all the systems, corresponding to the age the galaxy had when the system was born, and assume that each $t_{\text {born }}$ between 0 and the age of the galaxy $\left(t_{\mathrm{gal}} \sim 13.5 \mathrm{Gyr}\right)$ is equally likely, which corresponds to assuming a constant star formation rate.

Once the initial population has been generated, we use the latest version of the binary-star evolution (BSE) code from Hurley et al. (2002) to evolve the systems. We have slightly updated the BSE code: the critical mass ratio for dynamically stable mass transfer when the primary is in the Hertzsprung gap was changed from 4.0 to 3.2 (Han et al. 2003), and the rate of angular momentum loss due to magnetic braking was multiplied by the normalization factor provided by Davis et al. (2008). Finally, we corrected a small mistake found by Robert Izzard (priv. comm.) ${ }^{1}$

The systems are evolved for $t_{\mathrm{evol}}=t_{\mathrm{gal}}-t_{\mathrm{born}}$, to obtain the current orbital and stellar parameters. We assume a CE efficiency of 0.25 based on the results of Zorotovic et al. (2010) and the same fraction of recombination energy is included to compute the binding energy of the envelope.

\subsection{Results}

After evolving the systems, we obtain more than $4 \times 10^{5}$ detached PCEBs consisting of a WD and a MS star or BD secondary. The mass and core composition of the WD clearly depend on the evolutionary stage of the primary star when the CE phase occurs.

If the primary fills its Roche lobe during the first giant branch (FGB), the core will be mainly composed of helium. After the envelope is expelled, the core will not be massive enough to ignite helium and will evolve into a low-mass $\left(<0.5 M_{\odot}\right)$, heliumcore WD (He WD).

If the CE phase occurs close to the tip of the FGB, the core of the primary may be massive enough to start helium burning following envelope ejection. The minimum core mass for which this occurs depends on the initial mass of the primary (see, e.g., Han et al. 2002). When helium ignites after envelope ejection, the star becomes a hot sdB star, i.e. a helium-core-burning star with a very thin $\left(<0.02 M_{\odot}\right)$ hydrogen envelope and a typical mass of $\sim 0.45-0.5 M_{\odot}$. It remains in this stage for $\sim 10^{8} \mathrm{yr}$ until helium is exhausted in the core. The low mass of the envelope means the star cannot ascend the asymptotic giant branch (AGB)

\footnotetext{
1 Equation (32) from Hurley et al. (2002), related to tidal effects in close binaries, was misspelled in the code. The correct equation is $f_{\text {conv }}=\min \left[1,\left(\frac{P_{\text {tid }}}{2 \tau_{\text {conv }}}\right)^{2}\right]$, while in the code it was written $f_{\text {conv }}=$ $\min \left[1,\left(\frac{\left(P_{\mathrm{tid}}\right)^{2}}{2 \tau_{\mathrm{conv}}}\right)\right]$.
}

and instead becomes a WD (for a recent review of sdB stars see Heber 2009).

Finally if the primary fills the Roche lobe on the AGB the core of the primary will be more massive $\left(>0.5 M_{\odot}\right)$, and it will become a carbon/oxygen-core WD (C/O WD) after the ejection of the envelope. Very massive primaries can also produce oxygen/neon-core WDs but for the sake of simplicity we here call all post-AGB WDs C/O WDs.

The BSE code does not distinguish between He WDs and sdB stars; i.e., all the systems that enter the CE phase when the primary is on the FGB are assumed to evolve into PCEBs with He WD primaries. However, based on their current and initial masses, we can identify which of these systems are actually SdB stars: those with a mass higher than the minimum core mass $\left(M_{\mathrm{c}}^{\mathrm{min}}\right)$ needed to ignite helium after the envelope is lost. To compute $M_{\mathrm{c}}^{\mathrm{min}}$ we here use the results from Han et al. (2002, their Table 1), assuming a Reimers' wind mass-loss law (with $\eta=1 / 4)$ and solar metallicity ${ }^{2}$.

We divide the PCEB systems into four types, depending on the type of primary star they contain:

i) He WD primary, i.e., systems where the primary filled the Roche lobe on the FGB and $M_{\mathrm{wd}}<M_{\mathrm{c}}^{\mathrm{min}}$;

ii) sdB primary, i.e., systems where the primary filled the Roche lobe close to the tip of the FGB with $M_{\mathrm{wd}} \geq M_{\mathrm{c}}^{\mathrm{min}}$, which happened less than $10^{8} \mathrm{yr}$ ago;

iii) post-sdB primary, i.e., like the latter but if the CE phase occurred more than $10^{8} \mathrm{yr}$ ago, which means that the primary has been a helium burning sdB but has already evolved into a WD;

iv) $\mathrm{C} / \mathrm{O} \mathrm{WD}$ primary, i.e., systems that entered the $\mathrm{CE}$ phase when the primary was on the AGB.

Owing to the short duration of the sdB phase, we expect to find very few systems with primaries in this stage.

It should be noticed that $\mathrm{sdB}$ stars can also be formed in binaries through stable mass transfer. However, their orbital periods are expected to be typically about two orders of magnitude larger than those of PCEBs (e.g., Podsiadlowski et al. 2008), so we do not take into account this formation scenario here.

\subsubsection{The predicted current PCEB population}

Table 3 summarizes the number of systems that are found in each of the four groups just described, as well as their average orbital parameters. The subscript "f" corresponds to the final (current) value. As expected, very few ( $2 \%)$ systems with sdB primaries are still in this stage. The sample is dominated by systems with $\mathrm{C} / \mathrm{O}$ WD primaries. The relative number of systems with each type of primary depends on the value assumed for the CE efficiency, but the shape of the distributions are not significantly affected (see, e.g., Willems \& Kolb 2004; Politano \& Weiler 2007). The resulting orbital period, primary mass, and secondary mass distributions of the predicted current PCEB population are shown in Fig. 1. The complete population of detached PCEBs is represented by the gray shaded histograms. Distributions separating different types of primaries stars are shown as color histograms. Since we have obtained a very low percentage of systems that still contain an sdB primary, we multiplied the number of systems in each bin by ten (in green).

2 Han et al. (2002) only computed $M_{\mathrm{c}}^{\text {min }}$ for two fixed values of metal-
licity (0.02 and 0.004). However, it can be seen from their Fig. 1 that the
difference is not dramatic for stars with initial masses below $\sim 1.8 M_{\odot}$, which is the case for almost all the systems we obtain (see our Fig. 2). 
Table 3. Current orbital properties of the simulated PCEB population separated by the type of primary star.

\begin{tabular}{lccccccc}
\hline \hline Primary & $N$ & Percent & $\bar{M}_{1, \mathrm{f}}$ & $\bar{M}_{2}$ & $\bar{q}_{\mathrm{f}}=\overline{\left(\frac{M_{2}}{M_{1, \mathrm{f}}}\right)}$ & $\bar{P}_{\mathrm{f}}$ & $\widetilde{P}_{\mathrm{f}}$ \\
& $\times 10^{4}$ & $\%$ & {$\left[M_{\odot}\right]$} & {$\left[M_{\odot}\right]$} & & {$[\mathrm{d}]$} & {$[\mathrm{d}]$} \\
\hline He WD & 11.79 & 26.06 & $0.41 \pm 0.02$ & $0.65 \pm 0.29$ & $1.57 \pm 0.71$ & $1.03 \pm 0.86$ & 0.78 \\
sdB & 0.14 & 0.31 & $0.46 \pm 0.01$ & $0.65 \pm 0.39$ & $1.42 \pm 0.86$ & $3.23 \pm 3.42$ & 2.01 \\
post-sdB & 6.87 & 15.18 & $0.46 \pm 0.01$ & $0.61 \pm 0.31$ & $1.34 \pm 0.69$ & $1.81 \pm 1.69$ & 1.32 \\
C/O WD & 26.45 & 58.45 & $0.59 \pm 0.12$ & $0.67 \pm 0.42$ & $1.15 \pm 0.69$ & $50.75 \pm 154.94$ & 2.54 \\
\hline
\end{tabular}

Notes. The last column gives the median value for $P_{\mathrm{f}}$, while all the others correspond to the means (see text for details).

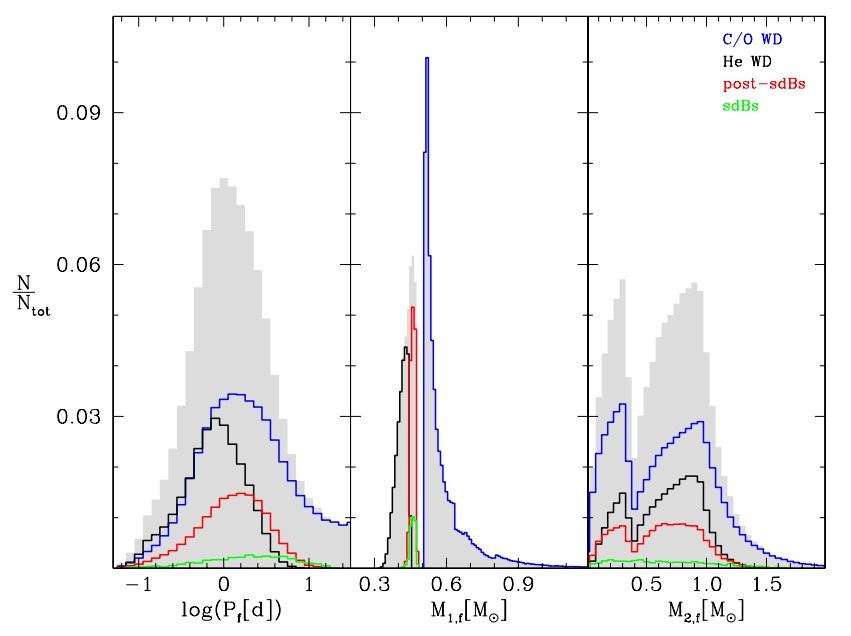

Fig. 1. Current orbital period (left), primary mass (middle), and secondary mass (right) distribution of the simulated PCEB population, separated according to the type of primary star they contain. The gray shaded histograms represent the entire population.

Systems in which the primary fills the Roche lobe at a more advanced evolutionary stage, which usually corresponds to a longer initial period, may also end up with a longer final period. This is clearly reflected in the average final periods listed in Table 3, where the larger orbital periods are for systems containing $\mathrm{C} / \mathrm{O}$ WDs, and is in perfect agreement with observations of WD+MS PCEBs (Zorotovic et al. 2011b). However, it should be noticed that the the mean value $\left(\bar{P}_{\mathrm{f}}\right)$ of the period distribution for PCEBs containing C/O WDs is strongly affected by a huge tail towards very long orbital periods. This tail corresponds to systems that fill the Roche lobe very late on the AGB and whose orbital separation decreases very slightly during CE evolution owing to the large amount of recombination energy available within the envelope. This is also reflected in the high value of the standard deviation for the mean value. Therefore, we also give in Table 3 the value of the median $\left(\widetilde{P}_{\mathrm{f}}\right)$, which is a better representation of the peak of the distribution and which still agrees with the results of Zorotovic et al. (2011b).

As expected, systems containing C/O WDs have $M_{1, \mathrm{f}}>$ $0.5 M_{\odot}$, while progenitors that filled their Roche lobe on the FGB have $M_{1, \mathrm{f}}<0.5 M_{\odot}$ separated by a small gap. The lower boundary of this gap is given by the maximum core mass that a giant star can have at the end of the FGB $\left(\sim 0.48 M_{\odot}\right)$. The upper boundary appears because the radius of the star at the beginning of the AGB is smaller than at the end of the FGB. The minimum WD mass for a star that filled its Roche lobe during the AGB is given by the core mass at which the radius first exceeds the maximum FGB radius. This happens for core
Table 4. Initial primary mass, mass ratio, and orbital separation of the simulated PCEBs with the different primary types previously defined.

\begin{tabular}{lcccc}
\hline \hline Primary & $\bar{M}_{1, \mathrm{i}}$ & $\bar{q}_{\mathrm{i}}=\overline{\left(\frac{M_{2}}{M_{1, \mathrm{i}}}\right)}$ & $\bar{a}_{\mathrm{i}}$ & $\bar{a}_{\mathrm{i}}$ \\
& {$\left[M_{\odot}\right]$} & & {$\left[R_{\odot}\right]$} & {$[\mathrm{AU}]$} \\
\hline He WD & $1.20 \pm 0.15$ & $0.53 \pm 0.23$ & $238 \pm 53$ & $1.11 \pm 0.25$ \\
sdB & $1.32 \pm 0.23$ & $0.48 \pm 0.26$ & $361 \pm 51$ & $1.68 \pm 0.24$ \\
post-sdB & $1.39 \pm 0.19$ & $0.45 \pm 0.22$ & $358 \pm 45$ & $1.67 \pm 0.21$ \\
C/O WD & $2.08 \pm 0.93$ & $0.34 \pm 0.21$ & $699 \pm 356$ & $3.25 \pm 1.65$ \\
\hline
\end{tabular}

masses $\sim 0.51 M_{\odot}{ }^{3}$. The mass distributions of He WDs and sdB (or post-sdB) stars overlap slightly, because the minimum core mass to ignite helium depends on the initial mass, but He WDs are in general slightly less massive than sdBs.

The distribution of companion masses is similar for all types of PCEBs, which is also consistent with the observations. There are more and more systems with increasing secondary masses, with a steep decline at $M_{2} \sim 0.35 M_{\odot}$, i.e. at the boundary for fully convective secondaries. According to the disrupted magnetic braking theory, systems with more massive secondaries suffer from angular momentum loss due to magnetic braking, in addition to gravitational radiation, and hence spend less time in the detached phase. This effect has been predicted theoretically by Politano \& Weiler (2007) and observationally confirmed by Schreiber et al. (2010). As expected, the orbital parameters for systems with $\mathrm{sdB}$ and post-sdB primaries are similar, with systems with a post-sdB primary having slightly shorter orbital periods since they have had more time to evolve towards shorter periods after the CE phase.

\subsubsection{Relating the initial separation and final system parameters}

In the context of detecting circumbinary planets around PCEBs, the initial parameters of the systems, and their relation with the current parameters is crucial.

One of the basic assumptions for the CE phase is that the mass of the secondary star remains constant, therefore the initial secondary masses are almost identical to the current secondary masses. The distributions of initial primary masses and initial separations are shown in Fig. 2, and the mean values are listed in Table 4. Again, systems with $\mathrm{sdB}$ and post-sdB primaries cover the same range of initial parameters. As in Fig.1 the number of systems with sdB primaries was multiplied by ten in Fig. 2 .

All the primaries that fill their Roche lobe on the FGB, i.e. the progenitors of PCEBs with $\mathrm{sdB}$ and He-core WD primaries, descend from low-mass stars $\left(M_{1, \mathrm{i}} \lesssim 1.8 M_{\odot}\right)$, while the

3 These limits slightly depend on the metallicity. 
M. Zorotovic and M. R. Schreiber: Origin of apparent period variations in PCEBs

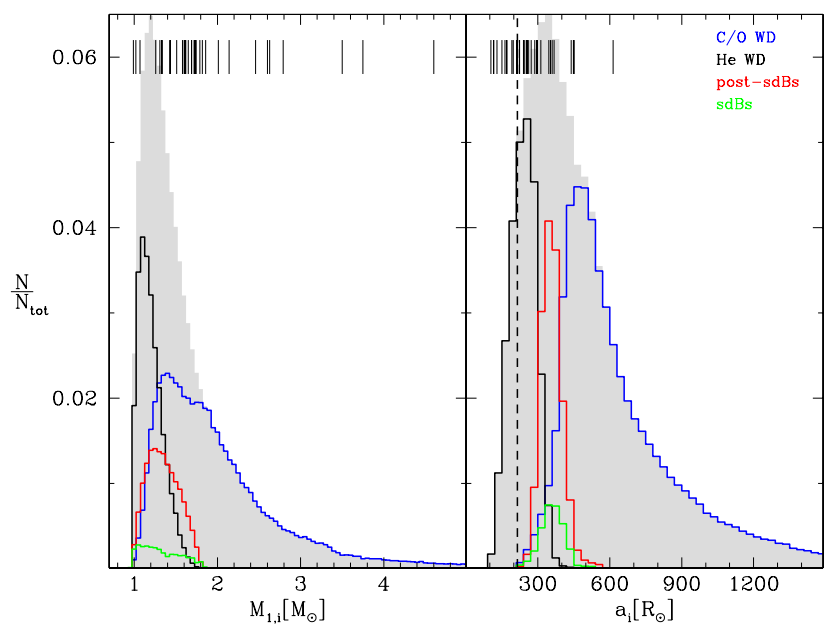

Fig. 2. Initial mass distribution of the primary (left) and initial separation (right) for the different types of PCEBs obtained with our simulation. The short vertical lines in the upper part of each panel correspond to the reconstructed values for the systems in Table 1 . The dashed vertical line corresponds to $1 \mathrm{AU}$.

progenitors of $\mathrm{C} / \mathrm{O}$ WDs are generally more massive and cover a wider range of masses.

Apparently, most PCEBs have formed from binaries with relatively small initial separations $a_{\mathrm{i}} \lesssim 1000 R_{\odot}$, i.e. $\$ 5 \mathrm{AU}$. The dashed vertical line in the right-hand panel of Fig. 2 represents 1 AU for comparison. PCEBs containing He WD primaries descend from very close binaries with $a_{\mathrm{i}}=100-400 R_{\odot}$, the progenitors of PCEBs with an sdB primary had initial separations of 200-500 $R_{\odot}$, and PCEBs with C/O WD primaries formed mostly from systems with initial separations $\gtrsim 500 R_{\odot}$. This is not surprising, because the radius of a giant star increases as the star evolves and the mass of the core grows, and therefore, larger separations are needed for the Roche lobe to be filled with a more massive core.

The relation between the initial binary separation and the initial and final stellar masses is shown in Fig. 3. There seems to be a relation with the initial primary mass and, more evidently, with the final WD mass. This is a consequence of the radius of a giant star depending almost exclusively on the core mass, i.e. on the mass of the future WD. This implies that the initial separation should also be larger in order to allow the star to evolve to this stage without filling the Roche lobe.

To compare the predictions of our simulations with the observed sample of eclipsing PCEBs, we use the reconstruction algorithm presented in Zorotovic et al. (2011a) to obtain the initial orbital parameters for all the systems in Table 1 with available periods and masses. The resulting values are included in Fig. 2 in the upper part of each panel ${ }^{4}$. The sample of observed eclipsing PCEBs is naturally biased towards short orbital periods, hence also slightly towards small initial binary separations of just a few $\mathrm{AU}$, but otherwise the properties of the simulated and reconstructed PCEBs are similar.

In summary, we found that PCEBs in general and the observed population of eclipsing PCEBs in particular descend from binaries with primary masses of $M_{1} \sim 1-3 M_{\odot}$, secondary masses from $0.1-1.0 M_{\odot}$ and initial separations of $a_{\mathrm{i}} \lesssim 5 \mathrm{AU}$.

\footnotetext{
4 We can only calculate the initial separations at the onset of the CE phase, which might differ from the actual initial separation due to, e.g., mass loss and tidal effects that may affect the orbital separation prior to CE evolution.
}

\section{Planet formation and planets around MS+MS binaries}

In the previous sections we have derived the properties of the MS+MS binary progenitors of PCEBs. In what follows we review what is known about planet formation and circumbinary planets around such MS+MS binaries. We start with a brief review of planet formation theories.

\subsection{Giant planet formation}

Giant planet formation theory has been developed early for the solar system and the pioneering works of the two still today competing models, i.e. the core-accretion model (Safronov 1969; Goldreich \& Ward 1973) and the disk-instability model (Cameron 1978), were published well before the first exoplanets were identified.

According to the disk-instability scenario, massive protoplanetary disks may fragment into dense cores that can contract further and form giant planets. Gravitational instabilities occur if Toomre's Q parameter (Toomre 1964) approaches unity and if the local cooling time is shorter than or equal to the orbital period at a given radius. Current models almost concordantly (to the best of our knowledge, the only exception is Boss 2012) show that such conditions might be present in massive young $(\$ 1 \mathrm{Myr})$ disks and only at distances from the central star $\gtrsim 20-100 \mathrm{AU}$ (e.g., Boley 2009; Cai et al. 2010). However, migration of the planet and scattering can redistribute the planetary orbits, and one can therefore not exclude relatively close planets also being formed due to disk instabilities.

According to the alternative scenario, the core-accretion model, larger protoplanetary cores are built by the accretion of planetesimals until the core is massive enough to accrete gas from the surrounding protoplanetary disk. The accretion of planetesimals is relatively slow, and the formation of giant planets according to the core-accretion models takes around four million years (Alibert et al. 2005). The strengths of the core-accretion model are that it naturally explains the planetmetallicity relation (Fischer \& Valenti 2005), that gas giants formed by core accretion are enriched due to the accretion of planetesimals as was been found to be the case in our solar system (Young 2003), and that rocky planets can form due to basically the same mechanism. Furthermore, population models based on the core-accretion scenario predict mass and semimajor axis distributions in agreement with observations (Ida \& Lin 2004; Alibert et al. 2005). Only very few massive planets around low-metallicity stars might be difficult to be explained within the core-accretion scenario (Mordasini et al. 2012). The core-accretion model is currently the favored formation model for most giant planets although giant planet formation due to disk instabilities cannot be excluded.

\subsection{Multiplicity and exoplanets}

Already almost two decades ago, Ghez et al. (1993) were the first to find a difference between the binary separation distributions of classical accreting T Tauri stars (CTTS) and disk-less weak-lined T Tauri stars (WTTS), in the sense that the fraction of WTTS to CTTS is increased for short projected separations $\$ 50$ AU. This finding has meanwhile been impressively confirmed by several studies using the unprecedented database provided by the Spitzer survey (e.g., Cieza et al. 2009), and similar results are found for the transition disk (Cieza et al. 2012) and debris disk systems (Rodriguez \& Zuckerman 2012). The lack 

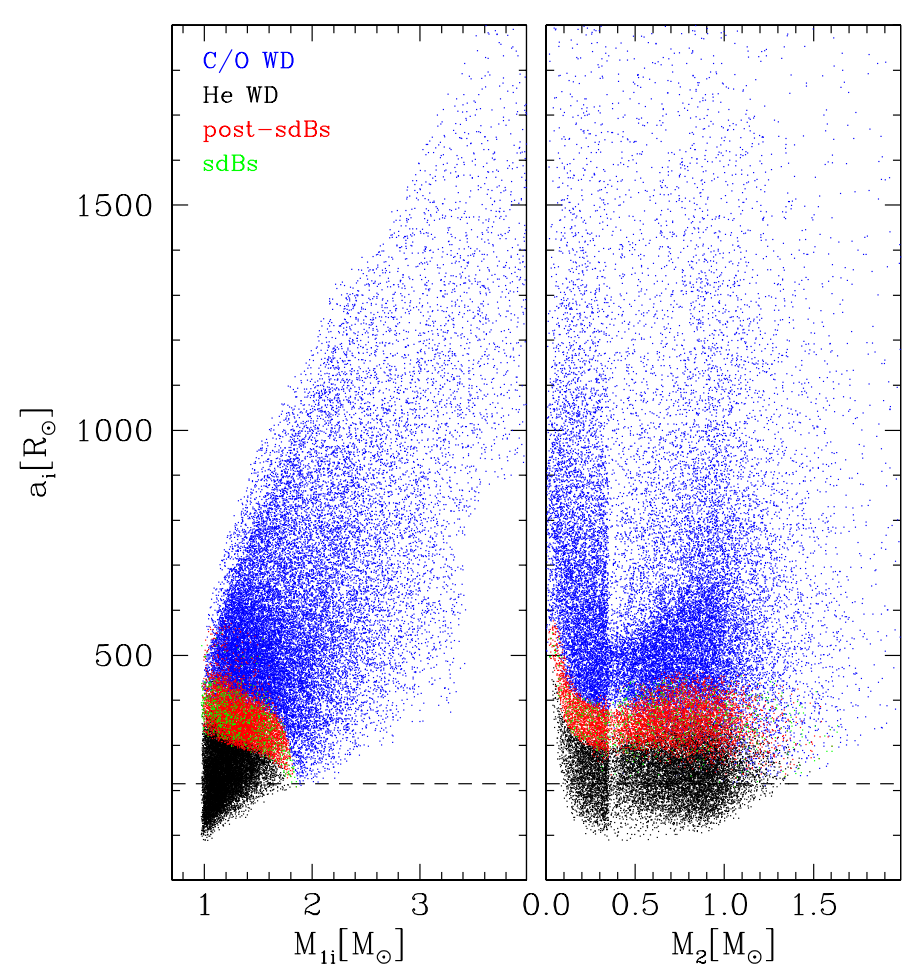

of binary stars with projected binary separations $\sim 1-100 \mathrm{AU}$ among samples of young stellar objects still hosting a disk may indicate a short lifetime of circumbinary disks around these close binaries.

Indeed, very recently, Kraus et al. $(2011,2012)$ have presented results of a high-resolution imaging study of the Taurus Auriga star-forming region of mostly solar type stars $(M \sim$ $\left.0.25-2.5 M_{\odot}\right)$ and compared the fraction of pre-MS stars hosting disks at different ages. They clearly show that the paucity of binaries among CTTS, transition disks, and debris disks is explained by the short lifetime of most circumbinary protoplanetary disks, i.e. $\$ 1 \mathrm{Myr}$. As this is short compared to the time scale of giant planet formation according to the core-accretion model, only a small fraction of these binaries should host circumbinary planets if core accretion is indeed the main channel for forming giant planets.

In addition to the short lifetimes of circumbinary disks around solar-type binary stars with projected separations $\gtrsim 1 \mathrm{AU}$, the search of planets around MS+MS binary stars within the field of NASA's Kepler mission revealed a low frequency of giant planets around even closer solar-type MS+MS binaries. The first planet around a pair of MS stars, Kepler-16, was found by Doyle et al. (2011). Later, Welsh et al. (2012) found two more planet transits-like events around the MS+MS binaries Kepler34 and Kepler-35, in a survey of 750 eclipsing binaries with periods ranging from 0.9 to 276 days, from Kepler's survey of MS+MS binaries. These three systems have binary periods longer than 20 days, while most of the systems observed by Kepler $(>80 \%)$ have shorter orbital periods. Considering that it is more likely to detect planets around short-period systems, this suggests a much lower rate of gas giant planets around the shorter period binaries. Based on geometric arguments, Welsh et al. (2012) infer a lower limit of $\sim 1 \%$ of circumbinary giant planets with periods around 100-200 days in close binaries ( $P=0.9-50$ days).

In summary, the low frequency of circumbinary planets complements the apparently short lifetimes of circumbinary disks

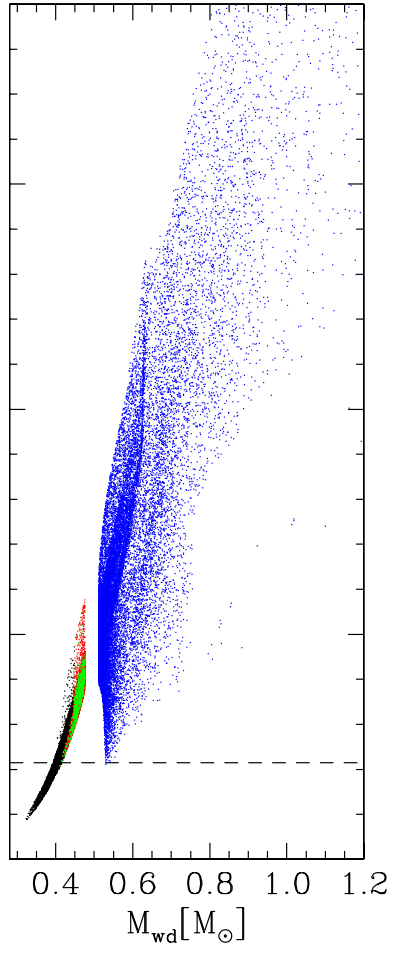

Fig. 3. Relation between the initial orbital separation and: initial primary mass (left), secondary mass (middle), and current mass of the primary (right). for projected separations of $\gtrsim 1 \mathrm{AU}$. We conclude that evidence for small numbers of circumbinary giant planets around close solar-type binary stars is growing. Such binary stars are the progenitors of PCEBs, so that the high frequency of apparent period variations observed in eclipsing PCEBs (see Sect. 2) is unlikely to be caused by giant planets that survived $\mathrm{CE}$ evolution. In fact, the only remaining possibility to explain the period variations observed in nearly all PCEBs due to such first-generation planets requires that nearly all first-generation circumbinary disks form giant planets at large separations that are not yet detectable with Kepler. Furthermore, these planets must form due to the fast disk-instability mechanism that is not in conflict with the short disk lifetimes mentioned above. While current observations cannot entirely exclude this possibility, it appears unlikely given that a flat extrapolation of the observed planet separation distribution to separations of 3-10 AU around single stars predicts that only $\sim 12$ per cent may host giant planets (Marcy et al. 2005).

\section{Discussion}

In the previous sections we obtained the following results

- From the 56 eclipsing, detached PCEBs with MS or BD secondaries, accurate eclipse time measurements covering more than around five years have been obtained for ten systems. All of these but AA Dor show apparent period changes that might indicate the presence of circumbinary planets.

- The progenitors of PCEBs are MS+MS binaries with initial separations of $\sim 0.5-5 \mathrm{AU}$, similar to those tested by Kraus et al. (2012) and Welsh et al. (2012).

- The reduced lifetime of circumbinary protoplanetary disks does not allow the frequent formation of circumbinary planets around close MS+MS binaries (Kraus et al. 2012) within the core-accretion model. This has been shown for projected separations $\sim 1-40 \mathrm{AU}$ and is likely to also be true for closer binaries (Welsh et al. 2012). 
These results suggest that the apparent period changes in PCEBs are not likely to be caused by circumbinary giant planets that formed in the circumbinary protoplanetary disk around the initial MS+MS binary. Instead, if the variations are indeed caused by circumbinary planets, the planets may have formed following CE evolution in a second-generation disk. The idea of second-generation planets goes back to the first exoplanet discovered around the pulsar PSR B1257+12. According to Tavani \& Brookshaw (1992), a circumbinary protoplanetary disk may have formed around the pulsar from evaporated material that did not leave the system. More recent models start from supernova fallback disks and can reproduce the observed eccentricities and masses of the planetary system around PSR B1257+12 (Hansen et al. 2009). It is therefore reasonable to suggest that also the claimed planets around PCEBs have a second-generation origin. In such a scenario, a protoplanetary disk must have formed following the CE ejection. This idea is supported by recent simulations of CE evolution indicating that envelope ejection can be incomplete, i.e. up to $\sim 10$ per cent of the ejected material may remain bound to the PCEB and is likely to form a circumbinary disk (Kashi \& Soker 2011; Ricker \& Taam 2012). If the apparent period changes in PCEBs are indeed caused by planets formed in such a second-generation protoplanetary disk, the planet formation process must proceed on a short time scale since the cooling age of the most convincing PCEB with planets, NN Ser, is just a million years. It must also be very efficient because nearly all PCEBs with accurate eclipse timings show apparent period variations. Below we discuss several options of second-generation planet formation and add a note of caution concerning the third-body interpretation.

\subsection{Second-generation disk instabilities}

The disk-instability model offers planet formation on short time scales that could solve the disagreement with the circumbinary planets around very young PCEBs such as NN Ser. However, in general, the disk-instability model still has to overcome serious problems, as recently discussed by Zhu et al. (2012). The orbital separations of the claimed planet detections around PCEBs disagree with the predictions of the disk instability model. Almost all the claimed planets are in orbits with $a \lesssim 10$ AU (see Table 2), while the conditions for forming planets according to the diskinstability scenario occur at distances $\gtrsim 20$ AU from the central star. Significant planet migration and/or scattering would thus be necessary. Furthermore, given the high fraction of PCEBs showing period variations would require that nearly all post-CE circumbinary disk are unstable and form giant planets. Although this scenario cannot be excluded, it is not clear why secondgeneration disks should be almost always unstable, fragment, and form giant planets, while disks around young single stars and MS+MS binaries seem to form giant planets in only about ten per cent of all cases. Therefore, second-generation disk instabilities do not appear to be the most promising solution.

\subsection{Second-generation rapid core accretion}

Planet formation according to the core-accretion model is more likely for higher metallicities (e.g. Fischer \& Valenti 2005), and therefore the production of massive elements during the AGB evolution might favor the formation of second-generation giant planets. Indeed, dusty disks surrounding binary post-AGB stars are a well known phenomenon. Waters et al. (1998) were the first to claim that such disks are similar to protoplanetary disks and may lead to the formation of planets. More recently, evidence has grown that dust-processing in these disks and in protoplanetary disks around young stars is probably very similar (Gielen et al. 2008). Moreover, a debris second-generation disk around an isolated neutron star has been detected (Wang et al. 2006). Furthermore, the second-generation giant planet formation process might be fast if the envelope that forms the second-generation disk is rich of heavy elements, especially $\mathrm{C}$ and $\mathrm{O}$, that trigger enhanced dust production and planet formation (Petigura \& Marcy 2011). Significantly enhanced dust-togas mass ratios may be able to explain both the high frequency of circumbinary planets and the short time scale of the secondgeneration planet formation required to explain the youth of the WD in NN Ser ( $\sim 1 \mathrm{Myr})$. Therefore, the second-generation, rapid core-accretion scenario seems to be a reasonable option to explain the observed apparent period variations.

Even better, based on the above, the second-generation disk model makes predictions that can be tested observationally. Enrichment of the envelope with heavy elements is well established as occurring at the end of the AGB but should be completely absent on the early FGB. As a result, second-generation disks around PCEBs with high-mass $\mathrm{C} / \mathrm{O}$ WD primaries should have much higher metallicities than around systems with lowmass He WD primaries and should therefore be found to more frequently host giant planets ${ }^{5}$.

A borderline case is PCEBs with sdB primary stars. Some authors claim that dust production may also occur very close to the tip of the FGB (Boyer et al. 2010), precisely where the progenitors of sdBs fill their Roche lobes. The evolution towards sdB stars is far from being completely understood. Late He-flashes may well lead to mixing in systems that experienced significant mass loss, but details are missing. We know neither the details of $\mathrm{CE}$ evolution nor pulsations or late He-flashes that may occur at the tip of the FGB. All we know for certain is that $\mathrm{sdB}$ stars are burning $\mathrm{He}$, which produces $\mathrm{C} / \mathrm{O}$, and one may speculate that this leads to enhanced metallicities in a potential second-generation disk around PCEBs with sdB primary stars.

The current data is inconclusive with respect to possible relations between the nature of the compact object and occurrence of circumbinary planets. There are nine PCEBs and three CVs with period variations attributed to circumbinary planets (see Table 1). Among the PCEBs, five of them contain an sdB primary, three a C/O WD, and only one a WD with $M_{\mathrm{wd}}<0.5$. For the last, RR Cae, apparent period variations have been only recently detected, and still need to be confirmed. Also, the mass of the WD is high enough to be either a He WD or a post-sdB star. In the case of CVs, all of them contain C/O WDs, which is not surprising given the observed mass distribution of WDs in CVs (Zorotovic et al. 2011a). These fractions agree with the secondgeneration disk scenario but are still far from providing clear constraints. Intense monitoring of a large sample of eclipsing PCEBs is required to test the predictions of second-generation planet formation.

\subsection{A hybrid first- and second-generation scenario}

Neptune-mass planets seem to be more frequent than giant Jupiter-like planets (Borucki et al. 2011), at least around single

5 However, this prediction is only based on metallicity, which might not be the only difference in the formation of $\mathrm{C} / \mathrm{O}$ and $\mathrm{He}$-core WDs that could affect potential planet formation in a second-generation circumbinary disk. Other factors, such as the expelled mass that is higher for AGB progenitors, may complicate the issue and dilute the prediction. 
stars. If this can be further confirmed, especially for planets around close MS+MS binary stars, it might be that secondgeneration planet formation is efficient because the remnants of lower mass planets survived $\mathrm{CE}$ evolution and serve as seeds for more massive planets. A similar scenario has been suggest by Perets (2010). As these planets already have a few Earth masses, the formation of a giant planet might be faster than for firstgeneration giant planets, and the short cooling age of the WD in some PCEBs is no longer a problem. The predictions of this scenario are a high fraction of low-mass planets around MS+MS binaries, which subsequently can grow due to the accretion of gas left by the CE phase. If this is the case, there should be no relation between the type of compact object in PCEBs and the frequency of circumbinary planets. While low-mass circumbinary planets around MS+MS binaries have indeed recently been detected (Orosz et al. 2012a,b), proper statistics of the frequency of low-mass circumbinary planets are not yet available.

\subsection{A note of caution: are we really detecting planets?}

As outlined in the introduction, pulsar timings led to detecting the first confirmed exoplanet orbiting the pulsar PSR B1257+12 (Wolszczan \& Frail 1992; Wolszczan 1994), so planets around compact binary stars do exist. However, one also has to keep in mind that the possible companion to PSR 1829-10 (Bailes et al. 1991) was retracted later (Lyne \& Bailes 1992).

The situation for PCEBs, and especially CVs, is similar. All we see are eclipse timing variations that can be reproduced well by assuming the existence of an orbiting circumbinary third body, but not a single set of eclipse times that confirms the parameters of a previously claimed planetary systems has been published so far. In contrast, some suggested planetary systems (e.g., Qian et al. 2010b, 2011) turned out to be dynamically unstable (Horner et al. 2011; Hinse et al. 2012) or drastically disagreed with more recent high-precision eclipse timings (Parsons et al. 2010b). It therefore remains an open question whether we indeed identified a new, large, and exciting population of circumbinary extrasolar planets or if perhaps an alternative process, such as the frequently proposed Applegate's mechanism (Applegate 1992) or a so far unknown process acting in deeply convective secondary stars might be responsible for at least some of the observed timing variations. In this context, AA Dor, a PCEB that may contain a BD companion, is extremely interesting because it is so far the only PCEB with continuous highprecision eclipse time measurements that does not show any signs of apparent period variations (Kilkenny 2011). One might therefore speculate that indeed the apparent period changes are somehow related to the existence of the convective secondary present in all PCEBs and CVs with claimed planet detections instead of being caused by circumbinary planets. Observations of eclipsing PCEBs with other types of secondary stars, i.e. with a WD or a BD companion (like e.g., SDSS J0820+0008), may give new insight into this alternative possibility.

\section{Conclusion}

By combining binary population models with recent observational and theoretical results for the formation of circumbinary giant planets, we have shown that the apparent period variations seen in virtually all close-compact binaries with good coverage ( $\gtrsim 5 \mathrm{yr}$ of mid-eclipse times with good accuracy) are unlikely to be explained by first-generation planets. The lifetimes of protoplanetary disks around MS+MS binaries are simply too short to form giant planets in most cases (Kraus et al. 2012), and the observed fraction of planets around MS+MS stars further confirms this (Welsh et al. 2012). The remaining options for explaining the observed period changes are either secondgeneration planet formation or alternative explanations that do not involve the existence of third and fourth bodies. We proposed observational experiments to test both hypothesis.

First, if not caused by circumbinary planets but the active secondary, the period variations should not be detected in close WD binaries with a second WD component or with a BD secondary. Second, if caused by second-generation planets, a clear relation between dust production in the envelope of the compact object progenitor and planet frequency is expected; i.e., planets should be more frequent around compact binaries with $\mathrm{C} / \mathrm{O}$ WDs than around those with a He-core WD primary. Continuous monitoring of all the eclipsing PCEBs listed in the appendix and also of all new systems that will be discovered will shed light on the origin of the observed apparent period changes in PCEBs.

Acknowledgements. M.Z. acknowledges support from comite mixto, Gemini/ Conicyt (grant 32100026) and CONICYT/FONDECYT/POSTDOCTORADO/ 3130559. MRS thanks the FONDECYT (project 1100782) and the Millennium Science Initiative, Chilean Ministry of Economy, Nucleus P10-022-F. We thank Steven G. Parsons for helpful discussions.

\section{References}

Alibert, Y., Mordasini, C., Benz, W., \& Winisdoerffer, C. 2005, A\&A, 434, 343 Almeida, L. A., \& Jablonski, F. 2011, in IAU Symp. 276, eds. A. Sozzetti, M. G. Lattanzi, \& A. P. Boss, 495

Almeida, L. A., Jablonski, F., \& Rodrigues, C. V. 2012a, ApJ, submitted [arXiv: 1210.3055]

Almeida, L. A., Jablonski, F., Tello, J., \& Rodrigues, C. V. 2012b, MNRAS, 423, 478

Almenara, J. M., Alonso, R., Rabus, M., et al. 2012, MNRAS, 420, 3017 Applegate, J. H. 1992, ApJ, 385, 621

Backhaus, U., Bauer, S., Beuermann, K., et al. 2012, A\&A, 538, A84

Bailes, M., Lyne, A. G., \& Shemar, S. L. 1991, Nature, 352, 311

Bailey, J., \& Cropper, M. 1991, MNRAS, 253, 27

Barlow, B. N., Kilkenny, D., Drechsel, H., et al. 2012, MNRAS, in press [arXiv: 1210.8146$]$

Beuermann, K., Thomas, H.-C., \& Schwope, A. 1988, A\&A, 195, L15 Beuermann, K., Hessman, F. V., Dreizler, S., et al. 2010, A\&A, 521, L60 Beuermann, K., Buhlmann, J., Diese, J., et al. 2011, A\&A, 526, A53 Beuermann, K., Breitenstein, P., Bski, B. D., et al. 2012a, A\&A, 540, A8 Beuermann, K., Dreizler, S., Hessman, F. V., \& Deller, J. 2012b, A\&A, 543, A138

Biermann, P., Schmidt, G. D., Liebert, J., et al. 1985, ApJ, 293, 303

Boley, A. C. 2009, ApJ, 695, L53

Borucki, W. J., Koch, D. G., Basri, G., et al. 2011, ApJ, 736, 19

Boss, A. P. 2012, MNRAS, 419, 1930

Boyer, M. L., van Loon, J. T., McDonald, I., et al. 2010, ApJ, 711, L99

Brinkworth, C. S., Marsh, T. R., Dhillon, V. S., \& Knigge, C. 2006, MNRAS 365,287

Bruch, A., \& Diaz, M. P. 1998, AJ, 116, 908

Cai, K., Pickett, M. K., Durisen, R. H., \& Milne, A. M. 2010, ApJ, 716, L176

Çakirli, O., \& Devlen, A. 1999, A\&A, 136, 27

Cameron, A. G. W. 1978, Moon and Planets, 18, 5

Çamurdan, C. M., Zengin Çamurdan, D., \& Ibanoğlu, C. 2012, New Astron., 17, 325

Charpinet, S., van Grootel, V., Reese, D., et al. 2008, A\&A, 489, 377

Cieza, L. A., Padgett, D. L., Allen, L. E., et al. 2009, ApJ, 696, L84

Cieza, L. A., Mathews, G. S., Williams, J. P., et al. 2012, ApJ, 752, 75

Clausen, D., Wade, R. A., Kopparapu, R. K., \& O'Shaughnessy, R. 2012, ApJ, 746,186

Dai, Z.-B., Qian, S.-B., Fernández Lajús, E., \& Baume, G. L. 2010, MNRAS, 409, 1195

Davis, P. J., Kolb, U., Willems, B., \& Gänsicke, B. T. 2008, MNRAS, 389, 1563

Davis, P. J., Kolb, U., \& Willems, B. 2010, MNRAS, 403, 179

de Kool, M., \& Ritter, H. 1993, A\&A, 267, 397

Doyle, L. R., Carter, J. A., Fabrycky, D. C., et al. 2011, Science, 333, 1602

Drake, A. J., Beshore, E., Catelan, M., et al. 2010, ApJ, submitted [arXiv: 1009. 3048]

Drechsel, H., Heber, U., Napiwotzki, R., et al. 2001, A\&A, 379, 893

Fischer, D. A., \& Valenti, J. 2005, ApJ, 622, 1102

Fleig, J., Rauch, T., Werner, K., \& Kruk, J. W. 2008, A\&A, 492, 565 
For, B.-Q., Green, E. M., Fontaine, G., et al. 2010, ApJ, 708, 253

Geier, S., Heber, U., Tillich, A., et al. 2010, Ap\&SS, 329, 91

Geier, S., Hirsch, H., Tillich, A., et al. 2011a, A\&A, 530, A28

Geier, S., Schaffenroth, V., Drechsel, H., et al. 2011b, ApJ, 731, L22

Geier, S., Classen, L., Brünner, P., et al. 2012, Low-mass stellar and substellar Companions to sdB stars, ASP Conf. Ser., 452, 153

Ghez, A. M., Neugebauer, G., \& Matthews, K. 1993, AJ, 106, 2005

Gielen, C., van Winckel, H., Min, M., Waters, L. B. F. M., \& Lloyd Evans, T. 2008, A\&A, 490, 725

Goldreich, P., \& Ward, W. R. 1973, ApJ, 183, 1051

Goździewski, K., Nasiroglu, I., Słowikowska, A., et al. 2012, MNRAS, 425, 930

Green, R. F., Richstone, D. O., \& Schmidt, M. 1978, ApJ, 224, 892

Guinan, E. F., \& Ribas, I. 2001, ApJ, 546, L43

Haefner, R. 1989, A\&A, 213, L15

Han, Z., Podsiadlowski, P., Maxted, P. F. L., Marsh, T. R., \& Ivanova, N. 2002, MNRAS, 336, 449

Han, Z., Podsiadlowski, P., Maxted, P. F. L., \& Marsh, T. R. 2003, MNRAS, 341, 669

Hansen, B. M. S., Shih, H.-Y., \& Currie, T. 2009, ApJ, 691, 382

Heber, U. 2009, ARA\&A, 47, 211

Hilditch, R. W., Kilkenny, D., Lynas-Gray, A. E., \& Hill, G. 2003, MNRAS, 344,644

Hinse, T. C., Lee, J. W., Goździewski, K., et al. 2012, MNRAS, 420, 3609

Hjellming, M. S., \& Taam, R. E. 1991, ApJ, 370, 709

Horner, J., Marshall, J. P., Wittenmyer, R. A., \& Tinney, C. G. 2011, MNRAS, 416, L11

Hurley, J. R., Tout, C. A., \& Pols, O. R. 2002, MNRAS, 329, 897

Ibanoglu, C., Keskin, V., Akan, M. C., Evren, S., \& Tunca, Z. 1994, A\&A, 281, 811

İbanoğlu, C., Çakırlı, Ö., Taş, G., \& Evren, S. 2004, A\&A, 414, 1043

İbanoğlu, C., Evren, S., Taş, G., \& Çakırlı, Ö. 2005, MNRAS, 360, 1077

Iben, I. J., \& Livio, M. 1993, PASP, 105, 1373

Ida, S., \& Lin, D. N. C. 2004, ApJ, 604, 388

Imamura, J. N., \& Steiman-Cameron, T. Y. 1998, ApJ, 501, 830

Kamiński, K. Z., Ruciński, S. M., Matthews, J. M., et al. 2007, AJ, 134, 1206

Kashi, A., \& Soker, N. 2011, MNRAS, 417, 1466

Kelley, N., \& Shaw, J. S. S. 2007, J. Southeastern Association Res. Astron., 1, 13

Kilkenny, D. 2011, MNRAS, 412, 487

Kilkenny, D., \& Koen, C. 2012, MNRAS, 421, 3238

Kilkenny, D., Hilditch, R. W., \& Penfold, J. E. 1978, MNRAS, 183, 523

Kilkenny, D., Hilditch, R. W., \& Penfold, J. E. 1979, MNRAS, 187, 1

Kilkenny, D., Hill, P. W., \& Penfold, J. E. 1981, MNRAS, 194, 429

Kilkenny, D., Marang, F., \& Menzies, J. W. 1994, MNRAS, 267, 535

Kilkenny, D., O’Donoghue, D., Koen, C., Stobie, R. S., \& Chen, A. 1997, MNRAS, 287, 867

Kilkenny, D., O'Donoghue, D., Koen, C., Lynas-Gray, A. E., \& van Wyk, F. 1998, MNRAS, 296, 329

Kilkenny, D., Keuris, S., Marang, F., et al. 2000, The Observatory, 120, 48

Kilkenny, D., van Wyk, F., \& Marang, F. 2003, The Observatory, 123, 31

Kiss, L. L., Csák, B., Szatmáry, K., Furész, G., \& Sziládi, K. 2000, A\&A, 364, 199

Klepp, S., \& Rauch, T. 2011, A\&A, 531, L7

Kraus, A. L., Ireland, M. J., Martinache, F., \& Hillenbrand, L. A. 2011, ApJ, 731,8

Kraus, A. L., Ireland, M. J., Hillenbrand, L. A., \& Martinache, F. 2012, ApJ, 745,19

Kroupa, P., Tout, C. A., \& Gilmore, G. 1993, MNRAS, 262, 545

Kruspe, R., Schuh, S., \& Traulsen, I. 2007, Inform. Bull. Variable Stars, 5796, 1

Krzeminski, W. 1984, IAU Circ., 4014

Kudritzki, R. P., Simon, K. P., Lynas-Gray, A. E., Kilkenny, D., \& Hill, P. W. 1982, A\&A, 106, 254

Kundra, E., \& Hric, L. 2011, Ap\&SS, 331, 121

Law, N. M., Kraus, A. L., Street, R. R., et al. 2011 [arXiv: 1101.0630]

Law, N. M., Kraus, A. L., Street, R., et al. 2012, ApJ, 757, 133

Lee, J. W., Kim, S.-L., Kim, C.-H., et al. 2009, AJ, 137, 3181

Liying, Z., \& Shengbang, Q. 2010, Ap\&SS, 329, 107

Lohsen, E. 1974, A\&A, 36, 459

Luyten, W. J. 1955, Luyten's Five Tenths. (Minneapolis: Lund Press)

Lyne, A. G., \& Bailes, M. 1992, Nature, 355, 213

Marcy, G., Butler, R. P., Fischer, D., et al. 2005, Prog. Theor. Phys. Suppl., 158, 24

Maxted, P. F. L., Marsh, T. R., Morales-Rueda, L., et al. 2004, MNRAS, 355, 1143

Maxted, P. F. L., O’Donoghue, D., Morales-Rueda, L., Napiwotzki, R., \& Smalley, B. 2007, MNRAS, 376, 919

Mayor, M., \& Queloz, D. 1995, Nature, 378, 355

Menzies, J. W., \& Marang, F. 1986, 118, 305
Mordasini, C., Alibert, Y., Benz, W., Klahr, H., \& Henning, T. 2012, A\&A, 541, A97

Müller, S., Geier, S., \& Heber, U. 2010, Ap\&SS, 329, 101

Nebot Gómez-Morán, A., Schwope, A. D., Schreiber, M. R., et al. 2009, A\&A, 495,561

Nelson, B., \& Young, A. 1970, PASP, 82, 699

Németh, P., Kiss, L. L., \& Sárneczky, K. 2005, IBVS

Niarchos, P. G., Gazeas, K. D., \& Manimanis, V. N. 2003, in Interplay of Periodic, Cyclic and Stochastic Variability in Selected Areas of the H-R Diagram, ed. C. Sterken, ASP Conf. Ser., 292, 129

O’Brien, M. S., Bond, H. E., \& Sion, E. M. 2001, ApJ, 563, 971

O’Donoghue, D., Koen, C., Kilkenny, D., et al. 2003, MNRAS, 345, 506

Orosz, J. A., Welsh, W. F., Carter, J. A., et al. 2012a, ApJ, 758, 87

Orosz, J. A., Welsh, W. F., Carter, J. A., et al. 2012b, Science, 337, 1511

Østensen, R., Oreiro, R., Drechsel, H., et al. 2007, HS 2231+2441: New Eclipsing sdB Binary of the HW Vir Type, ASP Conf. Ser., 372, 483

Østensen, R. H., Green, E. M., Bloemen, S., et al. 2010, MNRAS, 408, L51

Paczyński, B. 1976, in Structure and Evolution of Close Binary Systems, eds. P. Eggleton, S. Mitton, \& J. Whelan (Dordrecht: Kluwer), IAU Symp., 73, 75

Pandel, D., Cordova, F. A., Shirey, R. E., et al. 2002, MNRAS, 332, 116

Parsons, S. G., Marsh, T. R., Copperwheat, C. M., et al. 2010a, MNRAS, 402, 2591

Parsons, S. G., Marsh, T. R., Copperwheat, C. M., et al. 2010b, MNRAS, 407, 2362

Parsons, S. G., Marsh, T. R., Gänsicke, B. T., et al. 2011a, MNRAS, 1584

Parsons, S. G., Marsh, T. R., Gänsicke, B. T., \& Tappert, C. 2011b, MNRAS, 412, 2563

Parsons, S. G., Gänsicke, B. T., Marsh, T. R., et al. 2012a, MNRAS, in press [arXiv: 1211.0316]

Parsons, S. G., Marsh, T. R., Gänsicke, B. T., et al. 2012b, MNRAS, 420, 3281

Perets, H. B. 2010 [arXiv: 1001.0581$]$

Perryman, M. A. C., Cropper, M., Ramsay, G., et al. 2001, MNRAS, 324, 899

Petigura, E. A., \& Marcy, G. W. 2011, ApJ, 735, 41

Podsiadlowski, P., Han, Z., Lynas-Gray, A. E., \& Brown, D. 2008, in Hot Subdwarf Stars and Related Objects, eds. U. Heber, C. S. Jeffery, \& R. Napiwotzki, ASP Conf. Ser., 392, 15

Pogson, N. 1857, MNRAS, 17, 200

Politano, M., \& Weiler, K. P. 2006, ApJ, 641, L137

Politano, M., \& Weiler, K. P. 2007, ApJ, 665, 663

Polubek, G., Pigulski, A., Baran, A., \& Udalski, A. 2007, in 15th European Workshop on White Dwarfs, eds. R. Napiwotzki, \& M. R. Burleigh, ASP Conf. Ser., 372, 487

Potter, S. B., Romero-Colmenero, E., Ramsay, G., et al. 2011, MNRAS, 416, 2202

Pyrzas, S., Gänsicke, B. T., Marsh, T. R., et al. 2009, MNRAS, 394, 978

Pyrzas, S., Gänsicke, B. T., Brady, S., et al. 2012, MNRAS, 419, 817

Qian, S.-B., Dai, Z.-B., Zhu, L.-Y., et al. 2008, ApJ, 689, L49

Qian, S.-B., Dai, Z.-B., Liao, W.-P., et al. 2009a, ApJ, 706, L96

Qian, S.-B., Zhu, L.-Y., Zola, S., et al. 2009b, ApJ, 695, L163

Qian, S.-B., Liao, W.-P., Zhu, L.-Y., \& Dai, Z.-B. 2010a, ApJ, 708, L66

Qian, S.-B., Liao, W.-P., Zhu, L.-Y., et al. 2010b, MNRAS, 401, L34

Qian, S.-B., Zhu, L.-Y., Liu, L., et al. 2010c, Ap\&SS, 329, 113

Qian, S.-B., Liu, L., Liao, W.-P., et al. 2011, MNRAS, 414, L16

Qian, S.-B., Liu, L., Zhu, L.-Y., et al. 2012a, MNRAS, 422, L24

Qian, S.-B., Zhu, L.-Y., Dai, Z.-B., et al. 2012b, ApJ, 745, L23

Ramsay, G. 1994, Inform. Bull. Variable Stars, 4075, 1

Rauch, T. 2000, A\&A, 356, 665

Rebassa-Mansergas, A., Nebot Gómez-Morán, A., Schreiber, M. R., et al. 2012, MNRAS, 419,806

Ribeiro, T., Kafka, S., Baptista, R., \& Tappert, C. 2010, AJ, 139, 1106

Ricker, P. M., \& Taam, R. E. 2012, ApJ, 746, 74

Ritter, H., \& Kolb, U. 2003, A\&A, 404, 301

Robb, R. M., \& Greimel, R. 1997, Inform. Bull. Variable Stars, 4486, 1

Rodriguez, D. R., \& Zuckerman, B. 2012, ApJ, 745, 147

Rucinski, S. M. 2009, MNRAS, 395, 2299

Safronov, V. S. 1969, Evoliutsiia doplanetnogo oblaka, ed. V. S. Safronov

Schaffenroth, V., Geier, S., Heber, U., et al. 2011, in AIP Conf. Ser. 1331, eds. S. Schuh, H. Drechsel, \& U. Heber, 174

Schneider, J., Dedieu, C., Le Sidaner, P., Savalle, R., \& Zolotukhin, I. 2011, A\&A, 532, A79

Schreiber, M. R., \& Gänsicke, B. T. 2003, A\&A, 406, 305

Schreiber, M. R., Gänsicke, B. T., Rebassa-Mansergas, A., et al. 2010, A\&A, $513, \mathrm{~L} 7$

Schwarz, R., Schwope, A. D., Vogel, J., et al. 2009, A\&A, 496, 833

Schwope, A. D., Thomas, H. C., \& Beuermann, K. 1993, A\&A, 271, L25

Schwope, A. D., Hambaryan, V., Schwarz, R., Kanbach, G., \& Gänsicke, B. T. 2002, A\&A, 392, 541

Schwope, A. D., Horne, K., Steeghs, D., \& Still, M. 2011, A\&A, 531, A34 
Steinfadt, J. D. R., Bildsten, L., \& Howell, S. B. 2008, ApJ, 677, L113

Tas, G., Sipahi, E., Dal, H. A., et al. 2004, Inform. Bull. Variable Stars, 5548, 1 Tavani, M., \& Brookshaw, L. 1992, Nature, 356, 320

Tello, J., \& Jablonski, F. 2010, in Binaries - Key to Comprehension of the Universe, eds. A. Prša, \& M. Zejda, ASP Conf. Ser., 435, 93

Toomre, A. 1964, ApJ, 139, 1217

van den Besselaar, E. J. M., Greimel, R., Morales-Rueda, L., et al. 2007, A\&A, 466, 1031

Voges, W., Aschenbach, B., Boller, T., et al. 1999, A\&A, 349, 389

Vučković, M., Aerts, C., Östensen, R., et al. 2007, A\&A, 471, 605

Vučković, M., Østensen, R., Bloemen, S., Decoster, I., \& Aerts, C. 2008, Orbital effects in VLT-UVES Spectra of AA Dor and NY Vir, ASP Conf. Ser., 392, 199

Wang, Z., Chakrabarty, D., \& Kaplan, D. L. 2006, Nature, 440, 772

Waters, L. B. F. M., Cami, J., de Jong, T., et al. 1998, Nature, 391, 868

Webbink, R. F. 1984, ApJ, 277, 355

Webbink, R. F. 2008, in Astrophys. Space Sci. Lib. 352, eds. E. F. Milone,

D. A. Leahy, \& D. W. Hobill (Berlin: Springer), 233
Welsh, W. F., Orosz, J. A., Carter, J. A., Fabrycky, D. C., et al. 2012, Nature, 481,475

Willems, B., \& Kolb, U. 2004, A\&A, 419, 1057

Wils, P., di Scala, G., \& Otero, S. A. 2007, Inform. Bull. Variable Stars, 5800, 1 Wittenmyer, R. A., Horner, J., Marshall, J. P., Butters, O. W., \& Tinney, C. G. 2011, MNRAS, 1920

Wolszczan, A. 1994, Science, 264, 538

Wolszczan, A. 1997, in Planets Beyond the Solar System and the Next Generation of Space Missions, ed. D. Soderblom, ASP Conf. Ser., 119, 135 Wolszczan, A., \& Frail, D. A. 1992, Nature, 355, 145

Wood, J. H., \& Saffer, R. 1999, MNRAS, 305, 820

Young, R. E. 2003, New Astron. Rev., 47, 1

Zhu, Z., Hartmann, L., Nelson, R. P., \& Gammie, C. F. 2012, ApJ, 746, 110

Zorotovic, M., Schreiber, M. R., Gänsicke, B. T., \& Nebot Gómez-Morán, A. 2010, A\&A, 520, A86

Zorotovic, M., Schreiber, M. R., \& Gänsicke, B. T. 2011a, A\&A, 536, A42

Zorotovic, M., Schreiber, M. R., Gänsicke, B. T., et al. 2011b, A\&A, 536, L3 


\section{Appendix A: Notes on individual systems}

\section{A.1. Eclipsing $s d B+M S / B D$ PCEBs}

HW Vir is the prototype of HW Vir-like systems consisting of an sdB star with a late-type MS or BD companion. HW Vir systems have short periods $(\sim 2-3 \mathrm{~h})$, and the light curves show very sharp primary and secondary minima and a strong reflection effect. HW Vir itself was discovered by Menzies \& Marang (1986), who determined the period. Kilkenny et al. (1994) observed a change in the orbital period for the first time, which motivated further studies (e.g., Çakirli \& Devlen 1999; Wood \& Saffer 1999; Kiss et al. 2000; Kilkenny et al. 2000, 2003; İbanoğlu et al. 2004). Many possible explanations have been proposed and discussed, converging towards the existence of a third object with a long period and low mass. Qian et al. (2008) present new mid-eclipse times obtained from 2006 to 2008 that show some deviation from the sinusoidal fit proposed by Kilkenny et al. (2003) and İbanoğlu et al. (2004). They suggest a combination of cyclic variations plus a continuous decrease in the orbital period, which may reveal a fourth object with a long period. Lee et al. (2009) present 41 new mid-eclipse times taken from 2000 to 2008 and combine these with data from the literature. The Observed minus Calculated $(\mathrm{O}-\mathrm{C})$ diagram of the orbital period spanning more than 24 years shows a combination of two sinusoidal variations, probably produced by the presence of two substellar companions, plus a continuous period decrease that is too strong to be caused by gravitational radiation. Recently, Beuermann et al. (2012b) have published 26 new mid-eclipse times obtained between 2008 February and 2012 February, which deviate significantly from the Lee et al. (2009) prediction. They also find that the solution presented by Lee et al. (2009) is unstable and propose a new solution involving two companions to HW Vir: a planet and a BD or low-mass star.

HS 0705+6700 is an $\mathrm{sdB}+\mathrm{dM}$ binary discovered to be a detached short-period eclipsing system by Drechsel et al. (2001). Qian et al. (2009b) obtained 38 mid-eclipse times between 2006 and 2008, updated the ephemeris, and performed an $\mathrm{O}-\mathrm{C}$ diagram including 31 mid-eclipse times obtained from the literature since 2000 October (Drechsel et al. 2001; Niarchos et al. 2003; Németh et al. 2005; Kruspe et al. 2007). They detected cyclic variations that were attributed to the light-travel time effect produced by the presence of a third object. Qian et al. (2010c) present new mid-eclipse times taken in 2009, propose the existence of a continuous decrease in the orbital period, and derive a mass for the third body corresponding to a substellar object, probably a BD. Çamurdan et al. (2012) obtained new mideclipse times in 2010 December, which disagree with a longterm period decrease. They used a sinusoidal fit to adjust a third object in the system, which may be substellar or a very lowmass star, depending on the inclination. Recently, Beuermann et al. (2012a) have published new mid-eclipse times obtained between 2009 August and 2011 December, updated the ephemeris, and derive the parameters for a possible third body that seem to be more consistent with a substellar object.

$H S 2231+2441$ was discovered to be an eclipsing sdB with a low-mass, probably substellar, companion by Østensen et al. (2007), who determined the ephemeris and estimated the masses. Mid-eclipse times monitored since 2006 (Qian et al. 2010c) reveal a continuous decrease and cyclic variations in the orbital period. The cyclic oscillation suggests there is a third object in the system, and Qian et al. (2010c) estimate it may be a BD.

NSVS 14256825 was found to be an eclipsing $\mathrm{sdB}+\mathrm{dM}$ binary by Wils et al. (2007), who presented 19 primary mid-eclipse times obtained between 2007 June and September and derived the ephemeris and orbital parameters, which were recently updated by Almeida et al. (2012b). The system has also been monitored since 2006 by Qian et al. (2010c), whose preliminary results suggest a cyclic change in the orbital period. New mid-eclipse times obtained between 2010 September and 2011 October were published by Kilkenny \& Koen (2012). They found an increase in period, but a long baseline is needed to see whether this behavior is cyclic. In a parallel work, Beuermann et al. (2012a) present new mid-eclipse times obtained between 2009 July and 2011 October, which reveal an abrupt and continuous increase in the period after 2009. This is interpreted by the authors as the possible response to a third body in a highly elliptic orbit. In a very recent work, Almeida et al. (2012a) have reanalyzed the system including ten new mid-eclipse times between 2010 July and 2012 August and find that the variations observed in the $\mathrm{O}-\mathrm{C}$ diagram can be explained by two circumbinary giant planets.

$N Y$ Vir is an $\mathrm{sdB}+\mathrm{dM}$ binary whose eclipsing nature was revealed by Kilkenny et al. (1998). Subsequently, Kilkenny et al. (2000) present mid-eclipse times from 1996 to 1999, determined the ephemeris, and find no significant changes in the orbital period. However, Kilkenny (2011) combine the previous results with new mid-eclipse times taken between 2001 and 2010 and find a continuous period decrease, which is too high to be due to gravitational radiation. The strong decrease was also seen by Çamurdan et al. (2012), who have presented new mid-eclipse times from 2009 and 2011 and adjusted a downward parabola to the O-C diagram. Recently, Qian et al. (2012b) have combined the previous results with nine new mid-eclipse times obtained in 2011 May, updated the ephemeris, and propose that the $\mathrm{O}-\mathrm{C}$ diagram can be adjusted by a downward parabola plus a periodic variation produced by a planet orbiting the primary. They also suggest that the continuous period decrease may be part of a cyclic variation that may indicate the presence of a fourth object.

$2 M 1938+4603$ was found to be an eclipsing sdB+dM binary by Østensen et al. (2010), who derived the ephemeris and orbital parameters. The available mid-eclipse times only cover the period between 2008 June and 2010 May and therefore no conclusion about apparent period variations can be drawn yet.

NSVS 07826247 is the longest period $\mathrm{sdB}+\mathrm{dM}$ binary known to be eclipsing so far, discovered by Kelley \& Shaw (2007). Mideclipse times have been published by For et al. (2010, 2008 February to 2009 March), Liying \& Shengbang (2010, 2009 March to August), and Backhaus et al. (2012, 2011 February to October). No evidence of period variation has been found so far, but more observations are needed in order to discard variations.

$B U L-S C 16335$ was found to be an eclipsing system by Polubek et al. (2007), who suggest that it might be an HW Virlike system based on the appearance of the light curve. They derived the ephemeris and estimate some of the orbital parameters. Tello \& Jablonski (2010) rederived the orbital parameters and find disagreement with Polubek et al. (2007). The parameters are still poorly constrained and no mid-eclipse times are available so far.

$P G 1621+4737$ has been found in the course of the MUCHFUSS (massive unseen companions to hot faint underluminous stars from SDSS, Geier et al. 2011a) project by Geier et al. (2010), who determined the period and estimated that the companion to the sdB star is a very late-type MS star or a BD. The system parameters are not constrained and there are still no published mid-eclipse times.

SDSS J0820+0008 was discovered by Schaffenroth et al. (2011), who detected the short period radial velocity variations, 
observed the eclipses in the light curve indicating an HW Vir-like systems, and determined the period. The mass of the sdB primary covers a wide range of possible solutions, and the companion seems to be a BD. The ephemeris was derived by Geier et al. (2011b). Based on high-resolution spectroscopy with ESO-VLT/UVES Geier et al. (2012) confirm the substellar nature of the companion. They also find a significant shift in the system velocity with respect to the previous study, which may be produced by a third object in the system. However, more observations are needed.

ASAS 10232 was discovered by Schaffenroth et al. (2011), who determined the period and observed the primary eclipse in the light curve. The reflection effect, typical of HW Vir-like systems, is clearly observed. The orbital parameters were derived based on photometry and spectroscopy, and no mid-eclipse time has been published so far.

$A A D$ or is a short-period eclipsing binary containing an sdOB primary (Kilkenny et al. 1978). Although many subsequent investigations have been published (e.g., Kilkenny et al. 1979, 1981; Kudritzki et al. 1982; Rauch 2000; Hilditch et al. 2003; Fleig et al. 2008; Vučković et al. 2008; Rucinski 2009; Müller et al. 2010; Klepp \& Rauch 2011), the nature of the companion is still not clear, with some authors favoring a BD companion while others favor a very low-mass M star. Eclipse timings were studied by Kilkenny et al. (2000), who found no evidence of any period variations between 1977 and 1999. Kilkenny (2011) increased the baseline by including 13 new primary eclipse timings obtained between 2000 and 2010, updated the ephemeris, and confirmed the stability of the orbital period.

EC 10246-2707 has recently been reported as an eclipsing $\mathrm{sdB}+\mathrm{dM}$ binary by Barlow et al. (2012), who estimate the orbital parameters, derive the ephemeris, and present 49 mideclipse times covering 15 years between 1997 February and 2012 June. The $\mathrm{O}-\mathrm{C}$ diagram reveals no secular changes larger than $10^{-12} \mathrm{~s} \mathrm{~s}^{-1}$ in the period. However, the relatively low precision of the timings does not allow to rule out small-amplitude variations such as, e.g., those observed in NN Ser. Additional observations of eclipses with high precision are needed.

\section{A.2. Eclipsing WD+MS PCEBs}

NNSer was discovered to have deep eclipses by Haefner (1989), who classified the system as a DA+dM binary and measured the orbital period. Brinkworth et al. (2006) performed hightime-resolution photometry with ULTRACAM and detected a decrease in the orbital period that Applegate's mechanism fails to explain. They suggest it may be related to the presence of a third body. Qian et al. (2009a) propose a sinusoidal fit to the O-C diagram. Later, Parsons et al. (2010a,b) presented the result of eclipse observations performed with ULTRACAM since 2002, which disagree with the sinusoidal fit proposed by Qian et al. (2009a). However, they still consider that a third body may be the cause of the observed period changes. Beuermann et al. (2010) monitored the system during 2010 and combined their new mid-eclipse times with all the previously published times, reanalyzing those where it was necessary. They conclude that the large amplitude variations observed in the period can only be caused by a third body, and even suggest that the best model is obtained with two giant planets around the binary. However, the existence of the fourth body is still rather uncertain.

V471 Tau is a DA+dK2 system discovered to be eclipsing by Nelson \& Young (1970). Period variations have been observed for a long time (e.g., Lohsen 1974), and many possibilities were suggested in the past to explain the shape of the $\mathrm{O}-\mathrm{C}$ diagram (e.g., Ibanoglu et al. 1994; İbanoğlu et al. 2005; Guinan \& Ribas 2001; Kamiński et al. 2007), such as perturbations by a third body in a long-period orbit, apsidal motion due to a low orbital eccentricity, or even mass transfer. Recently, Kundra \& Hric (2011) have detected a change in the $\mathrm{O}-\mathrm{C}$ diagram trend. This allows them to exclude mass transfer and other models that predict a further increase in the $\mathrm{O}-\mathrm{C}$ value. After modeling the system, the authors find that the third component could be a BD or a very low-mass star, at a period of 33.2 years.

QS Vir was discovered in the Edinburgh-Cape faint blue object survey of high galactic latitudes (Kilkenny et al. 1997), where the eclipses revealed its binary nature. O'Donoghue et al. (2003) suggest that it is a hibernating CV, which was questioned by Ribeiro et al. (2010) and ruled out by Parsons et al. (2011b), who confirm that it is a detached system and not a hibernating $\mathrm{CV}$ based on high-resolution UVES spectra. Orbital period variations were analyzed by Qian et al. (2010b), who combined new and previously published mid-eclipse times, and propose that there is a giant planet and a continuous decrease in period due to magnetic braking. Parsons et al. (2010b) update the $\mathrm{O}-\mathrm{C}$ diagram by including ULTRACAM photometry and find strong disagreement with the fit of Qian et al. (2010b). They conclude that the decrease in orbital period is part of a cyclic variation that cannot be explained by Applegate's mechanism. A third body seems to be the most probable solution. Recently, Almeida \& Jablonski (2011) have presented new mid-eclipse times and suggest that the best fit in the $\mathrm{O}-\mathrm{C}$ diagram is obtained with a model with two circumbinary bodies, most likely a giant planet and a BD.

$R R C a e$ is a WD+dM binary discovered as a high proper motion object by Luyten (1955). The eclipses were first announced by Krzeminski (1984), and further observations of the eclipses were presented by Bruch \& Diaz (1998) and Maxted et al. (2007). The later paper updated the ephemeris and finds no evidence of variations in the orbital period on a long time scale ( 10 yr). Parsons et al. (2010b) performed ULTRACAM photometry for the system, obtained two new mid-eclipse times, and combined these with all the previous eclipse times available in order to study possible period variation. They obtained a roughly sinusoidal variation in the $\mathrm{O}-\mathrm{C}$ diagram, which can be explained via Applegate's mechanism. Qian et al. (2012a) have recently obtained six new mid-eclipse times that combined with those from the literature, show some evidence of a third object, a giant planet, and even possible evidence of a fourth companion. The last needs to be confirmed.

$D E C V n$ is a bright eclipsing $\mathrm{WD}+\mathrm{dM}$ binary discovered as an X-ray source by ROSAT (Voges et al. 1999). Recently, Parsons et al. (2010b) have obtained high-timeresolution photometry with ULTRACAM to obtain an accurate ephemeris. They combined their new mid-eclipse times with older times available in the literature (Robb \& Greimel 1997; van den Besselaar et al. 2007; Tas et al. 2004) in order to study possible period variations. However, only the ULTRACAM data are reliable, and there are still too few to analyze possible longterm period changes.

GK Vir was discovered by Green et al. (1978), who listed nine mid-eclipse times from 1975 April to 1978 February, and determined the orbital period. For more than twenty years there were no new eclipses observed. Between 2002 and 2007 Parsons et al. (2010b) observed seven primary eclipses with ULTRACAM and improved the ephemeris. After combining their points with those from Green et al. (1978), they observed a period increase and a slight variation in $\mathrm{O}-\mathrm{C}$ times. Drake et al. (2010) also published one mid-eclipse time for 
2005 April. Recently, Parsons et al. (2012b) have obtained a new high-precision mid-eclipse time on April 2010 using ULTRACAM, which shows a clear deviation from linearity. The magnitude of the period change is small and therefore can be caused by Applegate's mechanism or due to a third body in the system. More data is needed before the true cause of this period change can be established.

$R X J 2130.6+4710$ was discovered to have eclipses by Maxted et al. (2004), who determined the ephemeris and published three ULTRACAM mid-eclipse times obtained in 2002 and 2003. No new mid-eclipse times are available.

SDSS J0110+1326 and SDSS J0303+0054 were identified as eclipsing binaries by Pyrzas et al. (2009), who studied the eclipses between 2006 September and 2007 October, derived the ephemeris, and listed four and seven mid-eclipse times, respectively. Subsequently, Parsons et al. (2010b) analyzed accurate eclipses obtained during 2007 October using ULTRACAM and listed one and three new mid-eclipse times, respectively. They found some deviations from the ephemeris derived by Pyrzas et al. (2009) for SDSS J0110+1326, while the eclipses for SDSS J0303+0054 appear to be consistent in the two studies. However, given the short baseline and the large uncertainty in the mid-eclipse times derived by Pyrzas et al. (2009) further accurate observations are needed before long-term period changes can be explored. Recently, Backhaus et al. (2012) have presented six new mid-eclipse times for SDSS J0303+0054 between 2011 August and November, which increases the baseline for this system. The new eclipses are still consistent with linear ephemeris, however the authors still do not exclude long-term period variations.

SDSS J0857+0342 was first listed as an eclipsing system by Drake et al. (2010), who observed the regular eclipses as part of the Catalina Realtime Transient Survey and determined the short orbital period, which makes it the closest detached WD+dM binary. They published a mid-eclipse time for 2005 April. Recently, Parsons et al. (2011a) have presented nine new mid-eclipse times obtained between 2010 December and 2011 January with ULTRACAM, and updated the ephemeris that is so far consistent with the eclipse time listed in Drake et al. (2010). Independently, Backhaus et al. (2012) obtained seven more mid-eclipse times between 2010 November and 2011 October. Due to the uncertainty in Drake's eclipse, possible period changes cannot be discarded so far.

SDSS J1210+3347 was discovered to be eclipsing by Pyrzas et al. (2012), who obtained high-time-resolution photometry for nine eclipses between 2009 April and 2011 June using RISE on the Liverpool Telescope. They determined the orbital period and the ephemeris, however the mid-eclipse times still cover a short period of observation to study changes in the period.

SDSS J1212-0123 was listed as an eclipsing WD+dM binary by Nebot Gómez-Morán et al. (2009), who obtained six mid-eclipse times between 2007 January and 2008 May and derived the ephemeris. Parsons et al. (2012b) used ULTRACAM to obtained the first high-precision mid-eclipse in 2010 April, which was consistent with the mid-eclipse times from Nebot Gómez-Morán et al. (2009). They updated the ephemeris using the new accurate eclipse.

SDSS J1435+3733 is a partially eclipsing binary discovered by Steinfadt et al. (2008), who observed three eclipses in 2007 May and June. In a parallel study Pyrzas et al. (2009) independently identified the system, observed seven eclipses between 2007 February and May, and updated the ephemeris.

SDSS J1548+4057 was found to be eclipsing by Pyrzas et al. (2009), who derived the ephemeris and listed seven mid-eclipse times from 2008 May to July. Recently, Backhaus et al. (2012) presented six new mid-eclipse times between 2011 May and August, which are consistent with linear ephemeris but are still not enough to exclude long-term period variations.

CSS06653, CSS080502, CSS21616, CSS38094, CSS40190, CSS41631, and WD 1333+005 are all WD+dM binaries discovered to be eclipsing by Drake et al. (2010) as part of the Catalina Realtime Transient Survey. They only list one mid-eclipse time in 2005 April or May. Recently, Backhaus et al. (2012) present new mid-eclipse times obtained between 2011 January and October for all these systems, and derived the ephemeris again. The accuracy of Drake's eclipses is not good enough to confirm or exclude long-term period variations. However, most of the systems seem to be consistent with linear ephemeris (within a 2- $\sigma$ error), except for CSS06653 and WD 1333+005, which exhibit an increase in the observed period that is more than the expected error with respect to Drake's mid-eclipse times. More observations are necessary to confirm or discard these trends.

CSS07125, CSS080408, CSS09704, CSS09797, CSS21357, and CSS25601 were also discovered to be eclipsing WD+dM binaries by Drake et al. (2010) with one mid-eclipse time in 2005 April or May listed, except in the case of CSS21357 where no eclipse time is presented. No further studies are available for these systems.

PTFEB11.441, PTFEB28.235 and PTFEB28.852 are three eclipsing systems recently discovered by Law et al. (2012) during the PTF/M-dwarf survey (Law et al. 2011) for transiting planets around M-dwarfs. Only one mid-eclipse time is available for each system in 2010 August, September, and November, respectively.

KIC-10544976 is a WD+MS PCEB in the field of Kepler mission, recently published by Almenara et al. (2012). The authors list then mid-eclipse times: six in 2005, three in 2006, and one in 2008. Currently, the system has been continuously monitored by Kepler over two years, which makes it a good candidate to look for changes in the period in the near future.

SDSS J0821+4559, SDSS J0927+3329, SDSS J0946+2030, SDSS J0957+3001, SDSS J1021+1744, SDSS J1028+0931, SDSS J1057+1307, SDSS J1223-0056, SDSS J1307+2156, SDSS J1408+2950, SDSS J1411+1028 and SDSS J2235+1428 are all WD+MS PCEBs that were found to be eclipsing very recently by Parsons et al. (2012a) by correlating the SDSS WD+MS catalog (Rebassa-Mansergas et al. 2012) with Catalina Real-time Transient Survey light curves. Only one mideclipse time is available for each system.

\section{A.3. CVs with suspected planets}

UZFor is an eclipsing polar (or AM Her type CV) discovered by Beuermann et al. (1988). The orbital period has been analyzed by several authors (e.g., Ramsay 1994; Imamura \& Steiman-Cameron 1998; Perryman et al. 2001), who detected variations from linearity in the $\mathrm{O}-\mathrm{C}$ diagram. Dai et al. (2010) detected an increase in the orbital period and possible cyclical changes, which they suggest may be produced by a third lowmass object in the system. Recently, Potter et al. (2011) have combined new high-speed photometry spanning ten years with previous mid-eclipse times to obtain a baseline of 27 years. They find that the $\mathrm{O}-\mathrm{C}$ diagram is described best by a combination of two cyclic elliptical terms, probably due to the presence of two giant planets, and a secular variation.

$H U A q r$ is also an eclipsing polar discovered by Schwope et al. (1993) during the ROSAT All Sky Survey. Schwarz et al. (2009) updated the ephemeris and listed 72 eclipse egress 
times obtained between 1993 and 2007, revealing complex deviations from a linear trend in an $\mathrm{O}-\mathrm{C}$ diagram. Qian et al. (2011) combined these points with ten new eclipse egress times obtained between 2009 May and 2010 May, and find two cyclic variations in the $\mathrm{O}-\mathrm{C}$ curve and a long-term period decrease that cannot be explained by gravitational radiation or Applegate's mechanism. They propose that the cyclic variations observed are due to the presence of two giant planets, and the long-term period decrease may reveal a third planet. However, the proposed orbital parameters for the two possible planets have been questioned by Horner et al. (2011), who find the solutions extremely unstable on short time scales. Wittenmyer et al. (2011) used the same 82 eclipse egress times to fit different single- and double-planet models to the O-C diagram. They found that the best fits are obtained with two planets and that there is no need to invoke a third planet. Although their solutions are significantly different from the one given by Qian et al. (2011), the new parameters for the possible planets are still dynamically unstable, which casts some doubt on their existence. They speculate about other mechanisms being responsible for the observed variations, such as changes in the shape of the secondary star due to dynamo effect. However, in a very recent work, Goździewski et al. (2012) publish almost 60 new eclipse egress times with better accuracy. Combining these with reanalyzed previous data, the authors find that a single circumbinary companion gives the best explanation for the $\mathrm{O}-\mathrm{C}$ curve.

DP Leo was the first eclipsing polar discovered (Biermann et al. 1985). A decrease in the binary period was noticed by Schwope et al. (2002) and Pandel et al. (2002). Qian et al. (2010a) find a reversal of this trend, suggesting a sinusoidal variation that may be related to the presence of a giant planet. Beuermann et al. (2011) obtained accurate mid-eclipse times of the WD between 2009 March and 2010 February, updated the ephemeris, and combined their results with all the mid-eclipse times available by 2002, as published by Schwope et al. (2002). The data spanning more than 30 years since 1979 suggest there is a third body orbiting the binary, most likely a giant planet. 